\title{
Therapeutic outcome and related predictors of stereotactic body radiotherapy for small liver-confined HCC: A systematic review and meta-analysis of observational studies
}

\author{
Yanyan Long \\ Chongqing university cancer hospital \\ Yan Liang \\ University of California San Diego \\ Shujie Li \\ Chongqing University cancer hospital \\ Jing Guo \\ Chongqing university cancer hospital \\ Ying Wang \\ Chongqing university cancer hospital \\ Yan Luo \\ Chongqing university cancer hospital \\ YONGZHONG WU ( $\square$ cqmdwyz@163.com ) \\ Chongqing University https://orcid.org/0000-0001-8367-3347
}

Research

Keywords: Hepatocellular carcinoma (HCC), Stereotactic body radiotherapy (SBRT), Meta-analysis, Prognosis

Posted Date: November 18th, 2020

DOI: https://doi.org/10.21203/rs.3.rs-37331/v2

License: (c) (i) This work is licensed under a Creative Commons Attribution 4.0 International License. Read Full License

Version of Record: A version of this preprint was published at Radiation Oncology on April 8th, 2021. See the published version at https://doi.org/10.1186/s13014-021-01761-1. 


\section{Abstract}

Background and purpose: Stereotactic body radiotherapy (SBRT) is a promising ablative modality for hepatocellular carcinoma (HCC) especially for those with small-sized or early-stage tumors. This study aimed to synthesize available data to evaluate efficacy and explore related predictors of SBRT for small liverconfined HCC ( $\leq 3$ lesions with longest diameter $\leq 6 \mathrm{~cm}$ ).

Materials and methods: A systematic search were performed of the PubMed and Cochrane Library databases. Primary endpoints were overall survival (OS) and local control (LC) of small HCC patients treated with SBRT, meanwhile, to evaluate clinical parameters associated with treatment outcome by two methods including subgroup comparisons and pooled HR meta-analysis. The secondary endpoint was treatment toxicity including grade $\geq 3$ hepatic complication and radiation induced liver disease (RILD).

Results: After a comprehensive database review, 14 observational studies with 1238 HCC patients received SBRT were included in qualitative and quantitative analyses. Pooled 1-year and 3-year OS rates were 93.0\% (95\% confidence interval [Cl]: 88.0\%-96.0\%), and 72.0\% (95\% Cl: 62.0\%-79.0\%), respectively. Pooled 1year and 3-year LC rates were $96.0 \%$ (95\% Cl: 91.0\%-98.0\%), and 91.0\% (95\% Cl: 85.0\%-95.0\%), respectively. Subgroup comparisons regarding Child-Pugh class (stratified by CP-A percentage 100\%, 75-100\%, 50-75\%) showed there were statistically significant differences for both 1-year OS rate and 3-year OS rate ( $\mathrm{p}<$ 0.01 ), while that regarding number of lesions, pretreatment situation, age (median/mean age of 65), macrovascular invasion, tumor size, and radiation dose (median $\mathrm{BED}_{10}$ of $100 \mathrm{~Gy}$ ), there were no differences. In subgroup comparisons for LC rate, it showed number of lesions (1 lesion vs. 1-3 lesions) was significantly associated with 1 -year LC rate $(p=0.04)$, though not associated with 3 -year LC rate $(p=0.72)$. In subgroup comparisons categorized by other factors including pretreatment situation, age, CP-A percentage, macrovascular invasion, tumor size, and radiation dose, there were no significant differences for 1-year or 3-year LC rate. To further explore the association between CP class (A vs. B) and OS, the second method was applied by combining HR and 95\% Cls. Results indicated CP-A was predictive of better OS ( $\mathrm{p}=0.001)$ with pooled HR was 0.31 ( $95 \% \mathrm{Cls:} 0.11-0.88$ ), which was consistent with previous subgroup comparison results. Concerning adverse effect of SBRT, pooled rates of grade $\geq 3$ hepatic complications and RILD were $4.0 \%$ (95\% Cl: $2.0 \%-8.0 \%$ ) and $14.7 \%$ (95\% Cl: 7.4\%-24.7\%), respectively.

Conclusion: The study showed that SBRT was a potent local treatment for small liver-confined HCC conferring excellent OS and LC persisting up to 3 years, even though parts of included patients were pretreated or with macrovascular invasion. CP-A class was a significant predictor of optimal OS, while number of lesions might affect short term tumor control (1-year LC). Tumor size and radiation dose were not vital factors impacting treatment outcome for such smallsized HCC patients. Because of the low quality of observational studies and heterogeneous groups of patients treated with SBRT, further clinical trials should be prospectively investigated in large sample sizes.

\section{Introduction:}

Hepatocellular carcinoma (HCC), the most common primary liver malignancy, is regarded as the sixth most commonly diagnosed solid tumor, and the second leading cause of cancer-related death worldwide[1]. The prognosis and treatment options depend not only on the tumor stage but also on liver function and general condition of patients[2]. Liver transplantation, surgical resection, and local ablative therapies are applied with curative intent for patients with small early-stage HCC. However, liver transplantation is limited by organ availability and strict candidate selection criteria[3]. Surgical resection is commonly contraindicated due to presence of portal hypertension, cirrhotic liver with poor liver function or other medical comorbidities. Thus, in a large proportion of early-stage patients, local ablative therapies are the mainstay of treatment. These include radiofrequency ablation (RFA), microwave ablation (MWA), percutaneous ethanol injection (PEI), and external radiation therapy.

Classically, radiation therapy directed at the liver was of limited use, due to radiation-induced liver disease (RILD)[4]. However, along with the development of new delivery techniques, as well as new radiotherapeutic modalities, this has changed. Stereotactic body radiation (SBRT) has been pioneered by several centers worldwide as an alternative local ablative therapy for early small HCC[5-9]. For those tumors, SBRT precisely delivers high doses of radiation in just a few fractions conforming to the target volume with a low risk of radiation injury. The Asia-Pacific Primary Liver Cancer Expert meeting (APPLE), an association of liver cancer experts in the Asia-Pacific region, recommended application of SBRT for early-stage or small-sized HCC[10], especially if surgical resection or percutaneous ablative therapies are difficult, unfeasible, or rejected. This approach is also used as a salvage treatment for tumor recurrence after local radical therapies or for residual cancer after surgical resection or percutaneous ablative attempts. Nevertheless, most of the evidences based on observational studies. The role of SBRT in small HCC have not been well established due to the lack of high-level evidences. Correspondingly, it will lead to a lack of recognized predictors of treatment outcome (such as OS and LC) which are extremely important for optimal treatment planning.

Although randomized controlled trials provide the strongest evidence, they are time-consuming and labor-consuming. Clinical practices are often based on multiple smaller trials or clinical observations as well. Therefore, a meta-analysis of observational studies might be one of the best available options to evaluate the feasibility and efficacy of treatment and to provide useful information for clinical decision-making[11]. The aim of our study is to perform the first systematic review and meta-analysis of patient-specific outcomes of SBRT for small liver-confined HCC ( $\leq 3$ lesions with longest diameter $\leq 6 \mathrm{~cm}$ ) from a series of observational studies, and meanwhile, to comprehensively explore potential factors that can help clinicians in the therapeutic choice, determine stratification factors for future studies in this subset of patients.

\section{Materials And Methods】}

\section{Study protocol}

This study adhered to the Preferred Reporting Items for Systematic Reviews and Meta-Analysis (PRIMSA) guidelines. A systematic electronic search of PubMed and Cochrane Library databases was conducted on April 12, 2020, and re-run on April 30, 2020. We used the following search query: "( stereotactic 
body radiotherapy OR stereotactic ablative radiotherapy OR Cyber*Knife OR Gamma*Knife OR SBRT OR SABR ) AND (adenoma, hepatocellular OR Hepatocellular Carcinoma OR hepatic malignancy OR liver cancer OR hepatic neoplasm OR liver neoplasm)" to identify studies on SBRT for HCC patients published from 2000/01/01 to 2020/04/30 in English. Unpublished or other language studies were not included in the search. Detailed search query was shown in Supplementary Data 1-Part 1. The search terms were designed to find studies using SBRT or stereotactic ablative radiotherapy (SABR) to treat HCC, emphasizing clinical outcomes or adverse effect rather than technical perspectives.

\section{Selection criteria and data extraction}

After initial searching, it returned 444 results in total (346 in PubMed and 98 in Cochrane). Then studies were filtered to exclude duplicated studies, conference abstracts, reviews, letters, editorials, case reports, lab studies, and studies with irrelevant subjects using titles and citation. The remaining studies were reviewed by firstly reading abstract or patients character table, and/or next step comprehensively reading the full text to determine whether they fully met the inclusion criteria. The following inclusion criteria were used: (1) prospective and retrospective studies, reporting results of SBRT on small liver-confined HCC (13 lesions in liver with maximum single tumor diameter $\leq 6 \mathrm{~cm}$, no lymph node or extrahepatic metastasis), (2) provision of treatment outcome (OS or LC) or adverse effect; (3) inclusion of over 10 patients with HCC treated with SBRT; and (4) SBRT performed in < 10 fractions. In cases of multiple studies from one institution with overlapped patients, the following criteria were used, prioritized in numerical order, to determine inclusion: (1) study with the largest number of patients; (2) most recently published study. As this study involves different endpoints and different statistical methods to synthesize, we will properly sort the studies from same institution to different category according to the content of the paper. All in all, the aim is to calculate the same index without using repeated patients. Exclusion criteria were as follows: (1) unable to obtain full text; (2) SBRT was exclusively used as a bridge to liver transplantation; (3) combined with other anti-tumor treatment simultaneously (RFA, TACE, targeted therapy, immunotherapy, chemotherapy, et al.), but sequential therapy is allowed as long as there is at least 1-month interval; and (4) hypo-fractionated radiotherapy. All procedures to identify eligible studies were performed by two independent researchers (YYL and SJL). Any disagreement was resolved by discussion and mutual consent of the above two researchers and another researcher (YW).

The following data were obtained from original articles: (1) general information including authors, publication year, time of study, study design, country, number of patients and lesions, pre-treated or not, sex, and age; (2) clinical information including Child-Pugh class, ECOG (Eastern Cooperative Oncology Group) performance status, viral etiology, tumor vascular invasion, and tumor size; (3) treatment information and outcomes including SBRT dose, fractionation scheme, BED (Biologically Effective Dose), OS rate, LC rate, grade $\geq 3$ hepatic complication, and RILD; (4) predictors for OS or LC and related HR with $95 \%$ Cls if studies supplied or could be calculated from available numerical data using methods reported by Tierney et al.[12].

\section{Study definitions}

There, small liver-confined HCC was defined as 1-3 lesions in liver with maximum single tumor diameter $\leq 6 \mathrm{~cm}$, no lymph node or extrahepatic metastasis. We included one study[13] with tumor volume $\leq 100 \mathrm{cc}$ as diameter is calculated from volume, assuming tumor is spherical, which satisfied the criteria.

Treatment response was assessed using the Response Evaluation Criteria in Solid Tumors (RECIST) or modified RECIST (mRECIST) criteria on multiphase CT or MRI images performed after treatment, with very few unavailable[8, 9, 14, 15]. Local control (LC) was defined as absence of progression for target lesion (PTV). Overall survival (OS) and LC were estimated starting from the date of SBRT to the date of death or the final follow-up, and to the date of treated tumor progression or last follow-up, respectively, using the Kaplan-Meier method.

SBRT induced hepatic toxicity classification was according to Common Terminology Criteria for Adverse Events (CTCAE) for most studies, and very few based on Toxicity criteria of the Radiation Therapy Oncology Group (RTOG) and the European organization for research and treatment of cancer (EORTC)[7].

The definition of RILD was slightly different in different studies [7, 14-16], of which there are two types: classic RILD and non-classic RILD. Classic RILD was defined as anicteric hepatomegaly and ascites, or elevation of alkaline phosphatase more than twice above the upper limit of normal or baseline level, and non-classic RILD was defined as an elevation in the level of transaminases or bilirubin, which was graded according to CTCAE, or a decline in liver function measured by a worsening of CPS $\geq 2$ points.

\section{Quality assessment}

Because most (12/14) of the included studies were retrospective, the Newcastle-Ottawa Scale (NOS) [35] was applied to assess the quality of included studies by two investigators independently (SJL and JG). Studies with NOS scores of 7-9 were regarded as high-quality studies, and those with scores of 4-6 were considered medium-quality studies.

\section{Data synthesis and statistical methods}

The pooled estimated 1-year OS rate, 3-year OS rate, 1-year LC rate, 3-year LC rate, grade $\geq 3$ hepatic complication rate, and RILD rate were derived. Meta and Metafor Packages in R software were utilized to accomplish meta proportion analysis[17]. Raw proportion and other four methods (PLN, PLOGIT, PAS, PFT) to transform of raw proportion were performed for further analyses. By normal distribution test (Supplementary Data 1-Part 3) and carefully comparing the five methods, we chose the best Logit transformation of raw proportion (PLOGIT) for final analyses to increase validity. The PLOGIT is calculated as the log of raw proportion divided by one minus the raw proportion. $\operatorname{logit}(\mathrm{p})=\log (\mathrm{p} /(1-\mathrm{p}))$

The prognostic values of common clinical factors for treatment outcome (OS and LC) were explored through two different ways: (1) by subgroup comparisons, categorized by potential predictive factors using $\mathrm{R}$ meta proportion subgroup analysis (which is a method to explore heterogeneities originally);

(2) by combining HR and $95 \%$ Cls when there were at least three studies concerning same factor. If there were only two studies for certain predictor, we will not 
apply this method in order to avoid bias. The analyses were carried out using STATA software "metan" order. Multivariate data were preferable to univariate data if both were presented. However, univariate data were acceptable if multivariate results were not available. The pooled HRs were shown in the form of a forest plot. HR > 1 indicated poor survival or local control response when referring certain index, and vice versa.

As the included studies were performed at independent facilities using different radiation schedules, random effects models were adopted regardless of heterogeneity. Heterogeneity was considered to be present if the p value in Cochran's $Q$ test [36] was $<0.1$ and the $I^{2}$ value was $>50 \%$. Sensitivity analyses were conducted by excluding 1 study at a time and reanalyzing the remaining to test whether the results had changed substantially by any individual study. Publication biases were assessed using visual inspection of funnel plots and quantitatively assessed using Egger's test for the intercept [37]. A p value of $<0.05$ was considered statistically significant. All the statistical analyses were conducted using Stata version 15.1 (Stata Corporation, College Station, TX, USA), or R (R Core Team, 2019).

\section{Results:}

\section{Study characteristics}

An initial search of the two databases identified 444 studies. After exclusion of unqualified studies, finally, 14 studies[5-9, 13-16, 18-22] consisting of 1238 patients, fully meeting the inclusion criteria, were eligible for the present meta-analysis. The process of study recruitment was shown in Figure 1.

\section{Figure 1. Flowchart of study inclusion}

The majority of the included studies (12/14, 85.7\%) featured a retrospective design. Application of the NOS revealed all of the included studies to be of medium to high quality (Table 1). In 4 of 14 studies (28.6\%), only patients with small, single lesion were included, remaining studies included both single and multiple lesions $(\leq 3)$. And most studies $(11 / 14)$ included pretreated patients, with left 3 studies involving patients sole initially treated with SBRT. The age range of HCC patients was $30-90$ years and $73.9 \%$ of patients were male. All the patients were ECOG performance status $0-2$ and Child-Pugh class A-B respectively. In 3 studies, patients with vascular invasion were included, while most others were not containing such patients and 1 study did not mention it. We summarized studies whose median tumor sizes were available $[6,7,9,14,16,18-22]$ to get their overall median tumor size. It was $2.3 \mathrm{~cm}$ (range: $0.7-6.0$ ). Total dose of SBRT and fractionation schemes were found in most studies though quite heterogeneous. A biologically effective dose (BED) for the prescription dose was calculated using the standard linear-quadratic model (BED=D* $(1+d / \alpha / \beta)$, D means total dose, $d$ means dose per fraction). The calculation used the $\alpha / \beta$ ratio of 10 , to consider tumor radiobiology rather than that of normal tissues. It is important to optimize values of $\alpha / \beta$ in future studies, but currently it has still been common to use $\alpha / \beta=3$ Gy for some normal tissues and $\alpha / \beta=10$ Gy for some fast turnover tissues, such as tumors whose cell survival curves do not exhibit a pronounced shoulder[38]. The median value of all available median BED $_{10}$ estimates was 100Gy (range: 59.5 180.0Gy). Detailed information about the included studies are shown in Table 2 and Table 3.

Table 1. Assessment of study quality by Newcastle-Ottawa scale 


\begin{tabular}{|c|c|c|c|c|c|c|c|c|c|c|}
\hline \multirow[t]{2}{*}{ Study } & \multicolumn{4}{|c|}{ Selection } & \multicolumn{2}{|c|}{ Comparability } & \multicolumn{3}{|c|}{ Ascertainment of exposure/outcome } & \multirow[t]{2}{*}{ Score } \\
\hline & 1 & 2 & 3 & 4 & 5 & 6 & 7 & 8 & 9 & \\
\hline $\begin{array}{l}\text { Jing Sun }[7] \\
2019\end{array}$ & $\sqrt{ }$ & $\sqrt{ }$ & $\sqrt{ }$ & $\sqrt{ }$ & $x$ & $\sqrt{ }$ & $\sqrt{ }$ & $\sqrt{ }$ & $\sqrt{ }$ & 8 \\
\hline $\begin{array}{l}\text { Neehar D Parikh }{ }^{[8]} \\
2018\end{array}$ & $\sqrt{ }$ & $\sqrt{ }$ & $\sqrt{ }$ & $\sqrt{ }$ & $x$ & $x$ & $\sqrt{ }$ & $x$ & $\sqrt{ }$ & 6 \\
\hline $\begin{array}{l}\text { Baek Gyu Jun }{ }^{[18]} \\
2018\end{array}$ & $\sqrt{ }$ & $\sqrt{ }$ & $\sqrt{ }$ & $\sqrt{ }$ & $x$ & $x$ & $\sqrt{ }$ & $x$ & $\sqrt{ }$ & 6 \\
\hline $\begin{array}{l}\text { Baek Gyu Jun }{ }^{[14]} \\
2018\end{array}$ & $\sqrt{ }$ & $\sqrt{ }$ & $\sqrt{ }$ & $\sqrt{ }$ & $\sqrt{ }$ & $x$ & $\sqrt{ }$ & $\sqrt{ }$ & $\sqrt{ }$ & 8 \\
\hline $\begin{array}{l}\text { Yuri Jeong }{ }^{[16]} \\
2018\end{array}$ & $\sqrt{ }$ & $\sqrt{ }$ & $\sqrt{ }$ & $\sqrt{ }$ & $x$ & $\sqrt{ }$ & $\sqrt{ }$ & $\sqrt{ }$ & $\sqrt{ }$ & 8 \\
\hline $\begin{array}{l}\text { Ting-shi Su }{ }^{[19]} \\
2016\end{array}$ & $\sqrt{ }$ & $\sqrt{ }$ & $\sqrt{ }$ & $\sqrt{ }$ & $\sqrt{ }$ & $\sqrt{ }$ & $\sqrt{ }$ & $x$ & $\sqrt{ }$ & 8 \\
\hline $\begin{array}{l}\text { Atsuya Takeda }{ }^{[22]} \\
2016\end{array}$ & $\sqrt{ }$ & $\sqrt{ }$ & $\sqrt{ }$ & $\sqrt{ }$ & $x$ & $x$ & $\sqrt{ }$ & $\sqrt{ }$ & $\sqrt{ }$ & 7 \\
\hline $\begin{array}{l}\text { Marta Scorsetti }{ }^{[5]} \\
2015\end{array}$ & $\sqrt{ }$ & $\sqrt{ }$ & $\sqrt{ }$ & $\sqrt{ }$ & $\sqrt{ }$ & $\sqrt{ }$ & $\sqrt{ }$ & $x$ & $\sqrt{ }$ & 8 \\
\hline $\begin{array}{l}\text { Tomoki Kimura }{ }^{[20]} \\
2015\end{array}$ & $\sqrt{ }$ & $\sqrt{ }$ & $\sqrt{ }$ & $\sqrt{ }$ & $\sqrt{ }$ & $\sqrt{ }$ & $\sqrt{ }$ & $\sqrt{ }$ & $\sqrt{ }$ & 9 \\
\hline Kazue Shiozawa ${ }^{\left[{ }^{[9]}\right.} 2015$ & $\sqrt{ }$ & $\sqrt{ }$ & $\sqrt{ }$ & $\sqrt{ }$ & $x$ & $x$ & $\sqrt{ }$ & $x$ & $\sqrt{ }$ & 6 \\
\hline $\begin{array}{l}\text { Naoko Sanuki }{ }^{[21]} \\
2014\end{array}$ & $\sqrt{ }$ & $\sqrt{ }$ & $\sqrt{ }$ & $\sqrt{ }$ & $x$ & $x$ & $\sqrt{ }$ & $\sqrt{ }$ & $\sqrt{ }$ & 7 \\
\hline $\begin{array}{l}\text { Sang Min Yoon }{ }^{[6]} \\
2013\end{array}$ & $\sqrt{ }$ & $\sqrt{ }$ & $\sqrt{ }$ & $\sqrt{ }$ & $x$ & $x$ & $\sqrt{ }$ & $\sqrt{ }$ & $\sqrt{ }$ & 7 \\
\hline $\begin{array}{l}\text { Jinhong Jung }{ }^{[15]} \\
2013\end{array}$ & $\sqrt{ }$ & $\sqrt{ }$ & $\sqrt{ }$ & $\sqrt{ }$ & $\sqrt{ }$ & $\sqrt{ }$ & $\sqrt{ }$ & $\sqrt{ }$ & $\sqrt{ }$ & 9 \\
\hline $\begin{array}{l}\text { Jung Hyun Kwon }{ }^{[13]} \\
2010\end{array}$ & $\sqrt{ }$ & $\sqrt{ }$ & $\sqrt{ }$ & $\sqrt{ }$ & $\sqrt{ }$ & $\sqrt{ }$ & $\sqrt{ }$ & $\sqrt{ }$ & $\sqrt{ }$ & 9 \\
\hline
\end{tabular}

For cohort studies: 1, truly representative of exposed cohort; 2, non-exposed cohort drawn from the same community; 3, ascertainment of exposure; 4, outcome of interest not present at start; 5 , cohorts comparable on basis of tumor stage (BCLC/UICC), or tumor size, or Child-Pugh class/score according to different research endpoint (tumor stage was used if studies mainly on treatment outcome OS, or tumor size was used if studies mainly on treatment outcome LC, or $\mathrm{CP}$ class/score was used if studies only about toxicity) $; 6$, cohorts comparable on other factors (for example, we use liver Dmean for SBRT toxicity, CP class/score for OS, radiation dose for LC); 7 , quality of outcome assessment; 8 , follow-up long enough for outcome to occur (median/mean FU $\geq 12$ months for adverse effect, and $\geq 24$ months for treatment outcome OS/LC); and 9, complete accounting for cohort.

Table 2. General and clinical information of the included studies 


\begin{tabular}{|c|c|c|c|c|c|c|c|c|c|c|c|c|}
\hline Authors & Year & $\begin{array}{l}\text { Time } \\
\text { of } \\
\text { study, } \\
\text { design }\end{array}$ & Country & $\begin{array}{l}\text { No. of } \\
\text { patients } \\
\text { (lesions) }\end{array}$ & $\begin{array}{l}\text { Inclusion } \\
\text { of } \\
\text { pretreated } \\
\text { patients }\end{array}$ & $\begin{array}{l}\text { Sex } \\
(M / F)\end{array}$ & $\begin{array}{l}\text { Median Age } \\
\text { \range】 }\end{array}$ & $\begin{array}{l}\mathrm{CP}- \\
\mathrm{A} \%\end{array}$ & ECOG & $\begin{array}{l}\text { Viral } \\
\text { etiology } \\
\text { (HBV+HCV } \\
\%)\end{array}$ & $\begin{array}{l}\text { Inclusion } \\
\text { of } \\
\text { vascular } \\
\text { invasion } \\
\text { patients }\end{array}$ & $\begin{array}{l}\text { Median/mei } \\
\text { longest } \\
\text { diameter } \\
\text { ¿range, cm } \otimes\end{array}$ \\
\hline $\begin{array}{l}\text { Jing Sun } \\
\text { [7] }\end{array}$ & 2019 & $\begin{array}{l}\text { 2011- } \\
2014, \\
\text { R }\end{array}$ & China & $\begin{array}{l}108 \\
(108)\end{array}$ & No & $80 / 28$ & $54(37-77)$ & $100 \%$ & $0-1$ & $89.8 \%$ & No & $2.3(0.7-4.9)$ \\
\hline $\begin{array}{l}\text { Neehar D } \\
\text { Parikh }{ }^{[8]}\end{array}$ & 2018 & $\begin{array}{l}\text { 2004- } \\
2011 \\
\text { R }\end{array}$ & USA & 32 (NA) & No & $20 / 12$ & $66(72,71)$ IQR & NR & NR & NR & No & $\leq 5.0$ \\
\hline $\begin{array}{l}\text { Baek Gyu } \\
\text { Jun [18] }\end{array}$ & 2018 & $\begin{array}{l}\text { 2011- } \\
2016, \\
\text { R }\end{array}$ & Korea & 85 (125) & Yes & $65 / 20$ & 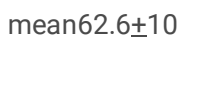 & $83.5 \%$ & NR & $68.2 \%$ & No & $2.23 \pm 1.17$ \\
\hline $\begin{array}{l}\text { Yuri } \\
\text { Jeong [16] }\end{array}$ & 2018 & $\begin{array}{l}2012- \\
2013, \\
\text { R }\end{array}$ & Korea & $\begin{array}{l}119 \\
(139)\end{array}$ & Yes & $97 / 22$ & $60(36-90)$ & $90.8 \%$ & $0-2$ & $87.4 \%$ & No & $1.7(\leq 6.0)$ \\
\hline $\begin{array}{l}\text { Ting-shi } \\
\text { Su [19] }\end{array}$ & 2016 & $\begin{array}{l}\text { 2009- } \\
2015 \\
\text { R }\end{array}$ & China & $\begin{array}{l}132 \\
(175)\end{array}$ & Yes & $110 / 22$ & 58(30-88\ & $86.4 \%$ & NR & $\begin{array}{l}90.2 \% \\
\text { (HBV only) }\end{array}$ & No & $3.0(1.1-5.0)$ \\
\hline $\begin{array}{l}\text { Marta } \\
\text { Scorsetti } \\
\text { [5] }\end{array}$ & 2015 & $\begin{array}{l}2010- \\
2014, \\
P\end{array}$ & Italy & $43(63)$ & Yes & $31 / 12$ & mean72(46-87ه & $53.5 \%$ & $0-2$ & $69.8 \%$ & Yes & $(\leq 6.0)$ \\
\hline $\begin{array}{l}\text { Tomoki } \\
\text { Kimura } \\
{[20]}\end{array}$ & 2015 & $\begin{array}{l}2008- \\
2013 \\
\text { R }\end{array}$ & Japan & $65(74)$ & Yes & $44-21$ & $73(49-90)$ & $86.2 \%$ & $0-1$ & $90.8 \%$ & No & $1.6(0.5-5.4)$ \\
\hline $\begin{array}{l}\text { Kazue } \\
\text { Shiozawa } \\
{[9]}\end{array}$ & 2015 & $\begin{array}{l}\text { 2011- } \\
2014, \\
\text { R }\end{array}$ & Japan & 35 (35) & No & $24 / 11$ & $\begin{array}{l}\text { mean75.1(55- } \\
89)\end{array}$ & $80.0 \%$ & $0-2$ & $77.1 \%$ & NR & $\begin{array}{l}2.86 \pm 1.15(1 \\
5.0)\end{array}$ \\
\hline $\begin{array}{l}\text { Naoko } \\
\text { Sanuki } \\
\text { [21] }\end{array}$ & 2014 & $\begin{array}{l}2005- \\
2012 \\
\text { R }\end{array}$ & Japan & $\begin{array}{l}185 \\
(185)\end{array}$ & Yes & $119 / 66$ & 74(40-89) & $85.4 \%$ & NR & $82.2 \%$ & Yes & $2.4 \llbracket 0.8-5.0 \rrbracket$ \\
\hline $\begin{array}{l}\text { Sang Min } \\
\text { Yoon [6] }\end{array}$ & 2013 & $\begin{array}{l}2007- \\
2009 \\
R\end{array}$ & Korea & 93 (103) & Yes & $75 / 18$ & $61(42-86)$ & $74.2 \%$ & NR & $87.1 \%$ & No & $2.0 \rrbracket \leq 6.0 \rrbracket$ \\
\hline $\begin{array}{l}\text { Jung } \\
\text { Hyun } \\
\text { Kwon [13] }\end{array}$ & 2010 & $\begin{array}{l}2004- \\
2007 \\
R\end{array}$ & Korea & 42 (NA) & Yes & $32 / 10$ & mean60.1 $1 \pm 10.9$ & $90.5 \%$ & $0-1$ & $85.7 \%$ & No & $\rrbracket \leq 6.0 \rrbracket^{*}$ \\
\hline $\begin{array}{l}\text { Baek Gyu } \\
\text { Jun [14] }\end{array}$ & 2018 & $\begin{array}{l}2011- \\
2015 \\
\mathrm{R}\end{array}$ & Korea & $\begin{array}{l}117 \\
(136)\end{array}$ & Yes & $86 / 31$ & 63(38-85】 & $76.1 \%$ & NR & $65.0 \%$ & No & $2.1(1.0-4.0)$ \\
\hline $\begin{array}{l}\text { Atsuya } \\
\text { Takeda } \\
\text { [22] }\end{array}$ & 2016 & $\begin{array}{l}2007- \\
2012 \\
P\end{array}$ & Japan & $90(90)$ & Yes & $58 / 32$ & 73(48-85】 & $91.1 \%$ & $0-2$ & $88.9 \%$ & Yes & $2.3(1.0-4.0)$ \\
\hline $\begin{array}{l}\text { Jinhong } \\
\text { Jung [15] }\end{array}$ & 2013 & $\begin{array}{l}2007- \\
2009 \\
\text { R }\end{array}$ & Korea & 92 (NA) & Yes & $74 / 18$ & 61(42-86区 & $73.9 \%$ & $0-2$ & $\begin{array}{l}75.0 \% \\
\text { (HBV only) }\end{array}$ & No & $(\leq 6.0 \otimes$ \\
\hline
\end{tabular}

Abbreviations: R: retrospective; P: prospective; NR: not reported

IQR, interquartile range;

*: diameter is roughly calculated from volume, assuming tumor is spherical.

Table 3. Treatment information and outcomes of the included studies 


\begin{tabular}{|c|c|c|c|c|c|c|c|c|c|c|}
\hline Authors & Year & $\begin{array}{l}\text { SBRT dose, } \\
\text { fractionation scheme }\end{array}$ & $\begin{array}{l}\mathrm{BED}_{10,} \text { Gy } \\
\text { Median(range) }\end{array}$ & $\begin{array}{l}\text { 1-year } \\
\text { OS }\end{array}$ & $\begin{array}{l}\text { 3-year } \\
\text { OS }\end{array}$ & $\begin{array}{l}\text { 1-year } \\
\text { LC }\end{array}$ & $\begin{array}{l}\text { 3-year } \\
\text { LC }\end{array}$ & $\begin{array}{l}\text { Grade } \geqq 3 \text { hepatic } \\
\text { complication (\%) }\end{array}$ & $\begin{array}{l}\text { RILD } \\
(\%)\end{array}$ & $\begin{array}{l}\text { Follow-up, } \\
\text { months }\end{array}$ \\
\hline Jing Sun ${ }^{[7]}$ & 2019 & 48-54Gy/5-8F & $\begin{array}{l}100(76.8- \\
102.6)\end{array}$ & $96.3 \%$ & $80.6 \%$ & $98.1 \%$ & $95.1 \%$ & $0 / 108 \varangle 0 \% \rrbracket$ & $\begin{array}{l}8 / 108 \\
\otimes 7.4 \% \bigotimes\end{array}$ & $42(6-77)$ \\
\hline $\begin{array}{l}\text { Neehar D } \\
\text { Parikh }{ }^{[8]}\end{array}$ & 2018 & NR & NR & $78.1 \%$ & / & I & I & I & I & $\begin{array}{l}\text { 16.2 (IQR: } \\
13.4-26.9)\end{array}$ \\
\hline $\begin{array}{l}\text { Baek Gyu } \\
\text { Jun }{ }^{[18]}\end{array}$ & 2018 & 40-60Gy/3-5F & $(72-180)$ & / & / & $91.1 \%$ & $89.9 \%$ & / & I & NR \\
\hline $\begin{array}{l}\text { Yuri Jeong } \\
\text { [16] }\end{array}$ & 2018 & 30-60Gy/3-4F & $\begin{array}{l}104.06(60- \\
180)\end{array}$ & $99.2 \%$ & $83.8 \%$ & $98.5 \%$ & $97.0 \%$ & $4 / 119$ (3.4\%区 & $\begin{array}{l}10 / 119 \\
\varangle 8.4 \% \rrbracket\end{array}$ & $\begin{array}{l}25.8(3.2- \\
36.8)\end{array}$ \\
\hline \multirow{2}{*}{$\begin{array}{l}\text { Ting-shi Su } \\
{[19]}\end{array}$} & \multirow[t]{2}{*}{2016} & $42-46 \mathrm{~Gy} / 3-5 \mathrm{~F}$ & \multirow[t]{2}{*}{ (77.3-162.5) } & \multirow[t]{2}{*}{$94.1 \%$} & \multirow[t]{2}{*}{$73.5 \%$} & \multirow[t]{2}{*}{$90.9 \%$} & \multirow[t]{2}{*}{ I } & \multirow[t]{2}{*}{$6 / 132(4.5 \%)$} & \multirow[t]{2}{*}{ / } & \multirow[t]{2}{*}{$21.0(3-76$} \\
\hline & & $28-30 \mathrm{~Gy} / 1 \mathrm{~F}$ & & & & & & & & \\
\hline \multirow{2}{*}{$\begin{array}{l}\text { Marta } \\
\text { Scorsetti }{ }^{[5]}\end{array}$} & \multirow[t]{2}{*}{2015} & $48-75 \mathrm{~Gy} / 3 \mathrm{~F}$ & \multirow[t]{2}{*}{$(57.6-262.5)$} & \multirow[t]{2}{*}{$77.9 \%$} & \multirow[t]{2}{*}{ I } & \multirow[t]{2}{*}{$85.8 \%$} & \multirow[t]{2}{*}{ I } & \multirow[t]{2}{*}{$7 / 43(16.3 \%)$} & \multirow[t]{2}{*}{ I } & \multirow[t]{2}{*}{$8(3-43)$} \\
\hline & & $36-60 G y / 6 F$ & & & & & & & & \\
\hline $\begin{array}{l}\text { Tomoki } \\
\text { Kimura }{ }^{[20]}\end{array}$ & 2015 & $48 \mathrm{~Gy} / 4 \mathrm{~F}$ & 105.6 & $92.0 \%$ & / & $100 \%$ & I & I & I & 26 \\
\hline $\begin{array}{l}\text { Kazue } \\
\text { Shiozawa }[9]\end{array}$ & 2015 & NR & NR & $95.2 \%$ & I & $97.1 \%$ & I & / & / & 12.6 \\
\hline $\begin{array}{l}\text { Naoko } \\
\text { Sanuki }{ }^{[21]}\end{array}$ & 2014 & 35 or $40 \mathrm{~Gy} / 5 \mathrm{~F}$ & $72(59.5$ or 72$)$ & $95.0 \%$ & $70.0 \%$ & $99.0 \%$ & $91.0 \%$ & I & I & $24(3-80)$ \\
\hline $\begin{array}{l}\text { Sang Min } \\
\text { Yoon [6] }\end{array}$ & 2013 & 30-60Gy/3-4F & $\begin{array}{l}104.06(60- \\
180)\end{array}$ & $86.0 \%$ & $53.8 \%$ & $94.8 \%$ & $92.1 \%$ & / & I & $\begin{array}{l}25.6(1.8- \\
55.4)\end{array}$ \\
\hline $\begin{array}{l}\text { Jung Hyun } \\
\text { Kwon [13] }\end{array}$ & 2010 & $30-39 G y / 3 F$ & $(60-89.7)$ & $92.9 \%$ & $58.6 \%$ & $72.0 \%$ & $68.0 \%$ & / & I & $\begin{array}{l}28.7(8.4- \\
49.1)\end{array}$ \\
\hline $\begin{array}{l}\text { Baek Gyu } \\
\text { Jun }{ }^{[14]}\end{array}$ & 2018 & 40-60Gy/3-5F & $(72-180)$ & I & / & I & I & / & $\begin{array}{l}29 / 117 \\
(24.7 \% \rrbracket\end{array}$ & $\begin{array}{l}22.5(3-56)- \\
\text { after SBRT }\end{array}$ \\
\hline $\begin{array}{l}\text { Atsuya } \\
\text { Takeda [22] }\end{array}$ & 2016 & 35 or $40 \mathrm{~Gy} / 5 \mathrm{~F}$ & (59.5 or 72) & I & I & I & I & $2 / 90(2.2 \%)$ & I & $\begin{array}{l}41.7(6.8- \\
96.2)\end{array}$ \\
\hline $\begin{array}{l}\text { Jinhong } \\
\text { Jung }{ }^{[15]}\end{array}$ & 2013 & 30-60Gy/3-4F & $(60-150)$ & I & / & I & / & $6 / 92(6.5 \%)$ & $\begin{array}{l}17 / 92 \\
\square 18.4 \% \bigotimes\end{array}$ & $\begin{array}{l}25.7(1.8- \\
55.4)\end{array}$ \\
\hline
\end{tabular}

Abbreviations: NR: not reported

\section{Treatment outcomes:}

\section{Pooled OS/LC rate and subgroup analysis}

Of 14 studies, 10 reported one-year OS rate[5-9, 13, 16, 19-21] and 6 reported three-year OS rate[6, 7, 13, 16, 19, 21]. The weighted mean values of 1-year OS, 3year OS rates were $92.8 \%$ (range: $77.9 \%-99.2 \%$ ), 71.9\% (range: $53.8 \%-83.8 \%$ ), respectively. One-year LC was available in 10 of 14 studies[5-7, 9, 13, 16, 18-21], ranging from $72.0 \%$ to $100 \%$ with a weighted mean of $94.6 \%$. Six of 14 studies $[6,7,13,16,18,21]$ reported 3 -year LC ranging from $68.0 \%$ to $97.0 \%$, with a weighted mean of $91.3 \%$. Treatment information and outcomes are summarized in Table 3.

Pooled rates using random effects analyses of 1-year, and 3-year OS were 93.0\% (95\% confidence interval [CI]: 88.0\%-96.0\%), and 72.0\% (95\% Cl: $62.0 \%-79.0 \%$ ), respectively (Figure $2 a, c)$. Significant heterogeneities among included studies were present in the two 0 S rates $\left(I^{2}>50 \%, p<0.1\right)$. Sensitive analyses were carried out by excluding 1 study at a time and reanalyzing the remaining and it showed the results had not changed substantially by any individual study (Figure 2b,d). In subgroup comparisons, differences between subgroups categorized by CP-A percentage (stratified by $100 \%$, $75-100 \%$, 50 $75 \%)$ were statistically significant for both 1-year OS rate $(p<0.01)$ and 3-year OS rate $(p<0.01)$, and the comparison could partly resolve statistical heterogeneity (Figure 3a,b). For subgroup comparisons categorized by other factors, including number of lesions (single lesion or inclusion of 2-3 lesions), pretreated or not, age (median/mean age of 65), macrovascular invasion, tumor size (stratified by median/mean longest diameter of $\leq 2 \mathrm{~cm}$ and $>2 \mathrm{~cm}$; and stratified by maximum diameter $\leq 5 \mathrm{~cm}$ and $5-6 \mathrm{~cm}$ ), and radiation dose (median $\mathrm{BED}_{10}$ estimates of $100 \mathrm{~Gy}$ ), no statistical differences were found among comparisons for 1-year OS and 3-year OS (Supplementary Figure 1,2).

Figure 2. Forest plots of pooled rates of treatment outcome and sensitive analyses. a-b, pooled 1-year OS rate and related sensitive analyses; c-d, pooled 3-year OS rate and related sensitive analyses; e-f, pooled 1-year LC rate and related sensitive analyses; g-h, pooled 3-year LC rate and related sensitive analyses.

Pooled rates using random effects analyses of 1-year LC, and 3-year LC were 96.0\% (95\% Cl: 91.0\%-98.0\%), and 91.0\% (95\% Cl: 85.0\%-95.0\%), respectively. Significant heterogeneities among included studies were present in the two LC rates as well $\left(I^{2}>50 \%, p<0.1\right)($ Figure 2 e,g). Sensitive analyses were carried out 
and showed the results had not changed substantially by any individual study (Figure $2 \mathrm{f}, \mathrm{h})$. In the subgroup comparisons regarding number of lesions ( 1 lesion or inclusion of 2-3 lesions), differences were statistically significant for 1-year LC rate ( $p=0.04)$, but not for 3-year LC rate ( $p=0.72)$ (Figure $3 c, d)$. It implied small HCC patients with less lesion might have better short-term local control when treated with SBRT, though in the long run, the effect was limited. For subgroup comparisons categorized by pretreatment situation, age (median/mean age of 65), CP-A percentage (stratified by 100\%,75-100\%,50-75\%), macrovascular invasion, tumor size (stratified by median/mean longest diameter of $\leq 2 \mathrm{~cm}$ and $>2 \mathrm{~cm}$; and stratified by maximum diameter $\leq 5 \mathrm{~cm}$ and $5-6 \mathrm{~cm}$ ), and radiation dose (median BED $_{10}$ estimates of 100Gy), no significant differences were found among comparisons for 1-year LC or 3-year LC (Supplementary Figure 3,4). In regarding to radiation dose, total dose $\left(\mathrm{BED}_{10} \leq 100 \mathrm{~Gy}\right.$ vs $\left.>100 \mathrm{~Gy}\right)$ was not a significant predictor for $\mathrm{LC}$ in this study, but the interpretation should be cautious because only two studies seems to be appropriate for subgroup analysis in lower total dose (Supplementary Figure 3B) and Jing Sun et al. [7] was borderline (The median total dose was $100 \mathrm{~Gy}$ ). Kwon et al. [13] showed the worst LC with the lowest total dose. In addition, the SBRT plan is performed differently by individual institutions. Sanuki et al. [21] optimized the plan so that the dose covering $95 \%$ of the PTV was more than the prescribed dose and Kwon et al. [13] prescribed the dose at the PTV with an isodose range of 70-85\% (median $80 \%)$. Hence, direct comparison of prescribed dose (BED 10 $\leq 100$ Gy vs $>100 \mathrm{~Gy}$ ) could have some limitations.

Figure 3. Forest plots of subgroup comparisons for treatment outcome. a, the impact of CP-class on 1-year OS rate; b, the impact of CP-class on 3-year OS; c, the impact of number of lesions on 1-year LC; $d$, the impact of number of lesions on 3-year LC.

\section{Predictors for OS by using pooled HR meta-analysis}

In order to further explore clinical predictors of OS and LC in small liver-confined HCC patients treated with SBRT, we applied the second method by combining $\mathrm{HR}$ and $95 \%$ Cls aim to identify parameters which can help clinicians in the therapeutic planning, and determine stratification factors for future studies in this subset of patients.

We screened 9 of 14 studies which involved various prognostic factors for treatment outcome (OS or LC) [5-7, 13, 14, 16, 19-21]. As there were very limited studies on LC rate, or some studies could not supply data of HR and $95 \% \mathrm{Cls}$, or there were less than 3 studies for certain factor, we excluded all such unqualified studies. Finally, only 5 studies $[7,13,14,16,19]$ concerning 4 predictors (CP class, tumor size, age, and sex) of OS were included for further analysis.

Association between CP class (A vs. B) and OS was presented in Figure 4a. The pooled HR using random effects analysis was 0.31 with range from 0.11 to 0.88 , which indicated CP-A was significantly predictive of better OS $(p=0.001)$. It is highly consistent with previous subgroup comparison results categorized by CP-A percentage (Figure 3a-b). Tumor size, age, and sex were not prognostic predictors of OS with the pooled HR of 1.11 (95\% Cl: $0.82-1.49$, P=0.165), 1.01 (95\% Cl: 0.99-1.04, P=0.673), and 0.70 (95\% Cl: 0.23-2.14, $\mathrm{P}=0.060)$, respectively (Figure 4b-d).

Figure 4. Forest plots of clinical parameters predicting OS in small HCC using SBRT. a, the impact of Child-Pugh class on OS; $b$, the impact of tumor size (including diameter and volume) on OS; c, the impact of age on OS; d, the impact of sex on OS.

\section{Hepatic complications}

The grade $\geq 3$ hepatic complications were available in 6 of 14 studies, while the results of RILD were available in only 4 of 14 studies. The weighted mean values of the above mentioned two indexes were $4.3 \%$ (95\% Cl: $0-16.3 \%)$ and $14.7 \%$ (95\% Cl: $7.4 \%-24.7 \%)$, respectively. And the pooled rates of the two indexes using random effects meta-analysis were $4.0 \%$ (95\% Cl: 2.0\%-8.0\%) and 15.0\% (95\% Cl: 8.0\%-22.0\%), respectively (Figure 5a,b). As the limited number of included studies, we did not do subgroup comparison or pooled HR meta-analysis to explore the impact factors.

Figure 5. Forest plots of pooled rates of hepatic complications in small HCC using SBRT. a, pooled rate of grade $\geq 3$ hepatic complications; b, pooled rate of RILD.

\section{Publication bias analysis}

Both visual inspections of funnel plots and Egger's test were carried out to test the publication biases quantitatively. Publication biases were identified from 1year LC rate studies ( $p=0.02014)$ while not from 3-year LC rate, 1-year OS rate, and 3-year OS rate studies ( $p>0.05)$ (Figure 6a-h).

Figure 6. Publication biases of included studies showing in funnel plots and Egger's test.

\section{Discussion】}

There we performed a meta-analysis of 14 studies encompassing 1238 patients who were treated with SBRT for small liver-confined HCC ( $\leq 3$ lesions with maximum single tumor diameter $\leq 6 \mathrm{~cm}$, no lymph node or extrahepatic metastasis). About the definition of small HCC, there is still no consensus in literature. In general, small sized HCC defined as $\leq 2 \mathrm{~cm}$ in EASL guideline, and fewer than three lesions with cumulative diameter $\leq 9 \mathrm{~cm}$ or single lesion up to $5 \mathrm{~cm}$ in MILAN criteria[10, 23]. But we still find some publications use the same definition as we do (longest diameter $\leq 6 \mathrm{~cm}$ and $\leq 3$ lesions). Expansion of the inclusion criteria in our study to $6 \mathrm{~cm}$ for longest diameter per lesion as SBRT candidates is due to active research field in some institutes from different countries especially in Korea[6,15,16], and results from multiple retrospective studies have proved its safety and good survival for SBRT among such tumor size $[5,13,15,16,20,24]$. In order to collect the actual clinical data, and distinguish it from the large lesion, we use "small" confined HCC there. Based on this criteria, our pooled results of 1-year OS, 3-year OS, 1-year LC, and 3-year LC rates were $93.0 \%$ (95\% Cl: 88.0\%-96.0\%), 72.0\% (95\% Cl: 62.0\%-79.0\%), 96.0\% (95\% Cl: $91.0 \%-98.0 \%$ ), and $91.0 \%$ (95\% Cl: $85.0 \%-95.0 \%)$, respectively. And pooled rates of grade $\geq 3$ hepatic complications and RILD were $4.0 \%$ (95\% Cl: $2.0 \%-8.0 \%$ ) 
and $14.7 \%$ (95\% Cl: $7.4 \%-24.7 \%)$, respectively. Despite inherent heterogeneity among observational studies, these results showed that SBRT is a feasible and safe local ablative modality with potent tumor control ability and survival benefit.

Small non-metastatic HCC is associated with good prognosis due to early or intermediate stage, and it is considered candidate for definitive treatment. According to European Association for the Study of the Liver (EASL) Clinical Practice Guidelines[25] and National Comprehensive Cancer Network (NCCN) Clinical Practice Guidelines in patients with early tumors, liver transplantation or tumor resection is preferable while ablation, arterially directed therapies or radiation therapy are considered an optional local treatment. In recommendations, SBRT is mostly considered as an alternative first-line therapy to the ablation/embolization techniques when these therapies have failed or are contraindicated, or just as a second- or multi-line salvage or palliative treatment after disease progression or recurrence if there is sufficient uninvolved liver. Here the rationale to perform this review and meta-analysis is based on the above rising incidence of SBRT treatment for small liver-confined HCC, no matter patients were initial or pretreated. Given that there are several treatment options for small HCC, accurate knowledge regarding patient survival after SBRT and the determination of significant factors that impact treatment outcome after SBRT are important for optimal treatment planning.

Liver transplantation was mostly restricted to a subgroup of patients meeting the Milan selection criteria (single tumors $\leq 5 \mathrm{~cm}$ in diameter or no more than three nodules $\leq 3 \mathrm{~cm}$ in diameter in patients with multiple tumors). 1-year OS rate after liver transplantation ranged from $84 \%$ to $90 \%$ and 3 -year OS rate was about $70 \%[23,26,27]$. Liver resection was associated with a 1 -year, 3-year, and 5-year survival rate of $85 \%-93.3 \%, 62 \%-76 \%$, and $51 \%-70 \%$ respectively, for selected patients with preserved liver function and early-stage $\mathrm{HCC}[23,27-30]$. In an ablative procedure, RFA is the most popular treatment option with previously published reports supporting its efficacy among early HCC patients[24, 31]. Results of some long-term study[31] showed that RFA as a first-line treatment for up to three HCCs with a maximum diameter of $5 \mathrm{~cm}$, whose cumulative incidence of local control rate was $85.5 \%$ at 5 years, and overall 5 -year survival rate was $67.9 \%$, respectively. And RFA alone for the treatment of smaller $(\leq 3 \mathrm{~cm}) \mathrm{HCCs}$, the treatment outcome was more optimal with 1-year, 3-year LC rate of $88.6 \%, 85.6 \%$, and 1-year, 3-year OS rate of $100 \%, 84.5 \%$, respectively[24]. In our group of patients ( $\leq 3$ lesions with longest diameter $\leq 6 \mathrm{~cm}$ ), the pooled 1-year and 3-year OS rates of SBRT were about $93.0 \%$ and $72.0 \%$, respectively, while pooled 1-year and 3-year LC rates were about $96.0 \%$ and $91.0 \%$, respectively. SBRT seemed to be non-inferior to surgery, and even had better LC rate than RFA although the included SBRT patients had worse prognostic criteria (larger tumor size including patients with 5-6cm diameter, pretreated, or some with macrovascular invasion). Such comparisons should, however, be made with caution. Both different selection criteria for patients qualified for recommended treatment modalities and varying quality of reporting in studies included in this review, may potentially introduce bias. Anyway, due to the strict indications or postoperative complications of surgery, and anatomical difficulties in approaching some lesions or heat sink effect of RFA, SBRT is a promising local modality with good tumor control ability and survival benefit. A recently published systematic review on SBRT for early-stage HCC showed[32] mean weighted OS across studies was $90.9 \%$ and $73.4 \%$ at 1 and 3 years, respectively, and mean weighted LC rate across studies was $94 \%$ and $93 \%$ at 1 and 3 years, respectively. Their results are slightly different to our results. But it should be noted that, their study is not truly including early small-sized HCC as the loose inclusion criteria. It involved patients with longest reported "median" diameter $<5 \mathrm{~cm}$, thus literally contained a part of patients with large tumors even up to $10 \mathrm{~cm}$ in diameter. In addition, C.H. Rim[33] conducted a meta-analysis including observational studies of SBRT for HCC with varied tumor size and stage published until April 23, 2018 encompassing 1950 patients. It showed pooled 1-, 2-, and 3-year OS rates were $72.6 \%, 57.8 \%$, and $48.3 \%$, respectively, and pooled 1-, 2-, and 3-year LC rates were $85.7 \%$, $83.6 \%$, and $83.9 \%$, respectively. As far as we know, there are no systematic reviews or meta-analyses available on treatment outcome of SBRT for truly small liver-confined HCC to date. Our results shed a light on it.

Determination of predictive factors that impact treatment outcome after SBRT is also crucial for optimal treatment scheme. Generally, for meta-analysis there are two methods to explore potential factors. One is subgroup comparison stratified by the certain factor, though the original purpose for such analysis is to figure out the heterogeneity. The other one is using the pooled HR and 95\% Cls when there were at least two studies focusing on the same factor. Comparatively, the former is much easier, while the latter is more stringent which requires the included studies to provide $\mathrm{HR}$ and $95 \% \mathrm{Cl}$, and it is also a more accepted method in meta-analysis for exploring impact factors. Literally, the two methods have been attempted in our study. Both subgroup analysis and pooled HR for prognostic factors analysis strongly proved CP-A class was significantly correlated with better OS compared to CP-B class. Nevertheless, other factors evaluated in this meta-analysis including radiation dose, whether pretreated or not, tumor size, tumor number, the presence of macroscopic vascular invasion, age, and sex did not impact treatment outcome of OS among those small-sized HCC patients. To evaluate predictors of LC, because of limited studies providing data of $\mathrm{HR}$ and $95 \% \mathrm{Cls}$, only subgroup analysis was carried out. Comparison regarding number of tumor lesions showed that cohort with single lesion patients compared to cohort with 1-3 lesions patients had better 1-year LC rate (but not 3-year LC). Other factors including radiation dose, whether pretreated or not, Child-Pugh's class, tumor size, tumor number and so forth did not impact treatment outcome of LC among those small-sized HCC patients. Of course, as mentioned earlier, we need to be cautious about the conclusion that radiation dose is not a significant predictor for LC, because direct comparison of prescribed dose $\left(\mathrm{BED}_{10} \leq 100 \mathrm{~Gy}\right.$ vs $\left.>100 \mathrm{~Gy}\right)$ could have some limitations. In addition, we compare the prognostic factors of SBRT to that of RFA, as RFA is a mature local procedure among early small-sized HCC. Lee et al. [31] indicated 5-year OS rate was 67.9\% with Child-Pugh class B as a significant predictive factors of RFA for poor survival $(R R=2.43, P=.011)$, which is similar to our results. However, it showed tumor size but not number of lesions as the only significant predictive factor of $L C(R R=2.13, P=.007)$. This is probably because the two local treatments are different in action mechanisms, and RFA is more likely subject to tumor size compared to radiotherapy. In addition, our results are inconsistent with the results of previous metaanalysis regarding the use of SBRT for HCC cases[33]. It showed subgroup comparison regarding tumor size but not CP class or number of tumor lesions had significant differences for 1- and 2-year OS rates and 1-, 2-, and 3-year LC rates. But subgroup comparisons regarding radiation dose, there were no difference for OS and LC, which is similar to our results. The reason for the differences is likely to the included patients in our studies are all small-sized cases while their study incorporated patients in various tumor size and stages. Among small HCC patients, tumor size or volume itself was not a vital factor to impact treatment outcome, and on the contrary, the liver function and number of lesions are strong impact factors.

Concerning adverse effect of SBRT, rates of grade $\geq 3$ hepatic complications and RILD were mostly mild (pooled rates of $4.0 \%$ and $14.7 \%$, respectively). However, one study[5] reported grade $\geq 3$ hepatic toxicity rate was $16.3 \%$. As limited studies supplied treatment-related toxicity and many did not distinguish 
between acute and late toxicities, it needs more prospective designed studies to validate. Considering the current pooled rates of complications and it might be caused by chronic liver disease itself, we support SBRT is a safe and feasible treatment for small HCC with CP-A/B class.

Of course, this study had several limitations. First and the most importantly, included studies for this meta-analysis were all observational studies which is controversial[34]. The variety of designs and populations among studies, and these differences might affect pooled estimates and inevitably brings about high heterogeneity. Though we applied random effects models for all the synthesized analysis, most results still had high heterogeneity ( $p<0.1$ and $I 2>50 \%$ ). We should treat the results with caution. Further clinical trials should be prospectively investigated in large sample sizes. Secondly, we assessed a bunch of clinical factors, such as Child-Pugh class, tumor size, tumor number, and so on, but with a simplified manner by subgroup analysis. Even though we tried two methods to analyzing survival impact parameters, the included studies for more convincing pooled-HR method were in minority. Thirdly, though there were 14 studies included in this study, when they were assigned to different research endpoints, there were relatively small number of studies under each endpoint. For example, analysis of 1-year OS and LC included 10 studies, but analysis of 3-year OS and LC only included 6 studies, and pooled HR meta-analysis for OS stratified by Child-Pugh class, tumor size, age, and sex included 3, 4, 3, 3 studies, respectively. The limited number of included studies will induce great bias and high heterogeneity. Fourthly, as we mentioned before, there were limited data about treatment-related toxicities, which needed further studies to validate. Finally, in included studies, only a small percentage of patients were treated with SBRT as an initial treatment. Remaining patients have previously undergone other locoregional or surgical treatments. In order to reduce the influence of previous treatment, though we set an at least 1-month interval between the two therapies, there might still exist some summed effect of the previous treatment on the observed effect in a patient. However, despite the limitations, we presented analyses based on the latest and most comprehensive data and such results provide detailed information about efficacy of SBRT for small HCCs and the predictors for treatment outcome.

\section{Conclusion}

Results of this review demonstrated SBRT was a potent local treatment for small liver-confined HCC conferring excellent OS and LC persisting up to 3 years, even though parts of included patients were pretreated or with macrovascular invasion. CP-A class was a significant predictor of optimal OS, while number of lesions might affect short term tumor control (1-year LC). Tumor size and radiation dose were not vital factors impacting treatment outcome for such smallsized HCC patients. Though the results are limited by the low quality of studies and heterogeneous groups of patients treated with SBRT, this provides a rationale for further studies applying SBRT for small HCCs as a first-line treatment or after other treatment, especially for those with single lesion and better CP class. Because of the low quality of observational studies and heterogeneous groups of patients treated with SBRT, we should treat the results with caution. But It provides a rationale for further clinical trials applying SBRT for small HCCs as a first-line treatment or after other treatment, especially for those with single lesion and better CP class. We hope it can be prospectively investigated in large sample sizes.

\section{Abbreviations:}

APPLE: the Asia-Pacific Primary Liver Cancer Expert meeting; BED: Biologically Effective Dose; Cl: confidence interval; CP: Child-Pugh; CTCAE: Common Terminology Criteria for Adverse Events; EASL: European Association for the Study of the Liver; ECOG: Eastern Cooperative Oncology Group; EORTC: the European organization for research and treatment of cancer; HCC: Hepatocellular carcinoma; LC: Local control; mRECIST: modified RECIST; MWA: Microwave ablation; NCCN: National Comprehensive Cancer Network; NOS: the Newcastle-Ottawa Scale; NR: Not reported; OS: Overall survival; PEl: Percutaneous ethanol injection; PRIMSA: the Preferred Reporting Items for Systematic Reviews and Meta-Analysis; RECIST: the Response Evaluation Criteria in Solid Tumors; RFA: Radiofrequency ablation; RILD: Radiation induced liver disease; RTOG: the Radiation Therapy Oncology Group; SBRT: Stereotactic body radiotherapy; TACE: Transcatheter arterial chemoembolization.

\section{Declarations:}

Ethics approval and consent to participate: Not applicable.

Consent for publication: Not applicable.

Availability of supporting data: All data generated or analysed during this study are included in this published article and its supplementary information files.

Competing interests: The authors declare that they have no competing interests.

Funding: This work was supported by grants from Chongqing scientific research institute performance incentive guidance project funded by Chongqing Science and Technology Foundation (cstc2018jxj130041), and Chongqing key project of major disease prevention and treatment technology funded by Chongqing Municipal Public Health Bureau (2019ZX002).

Authors' contributions: YYL helped design the work, searched the database and collected some data, did parts of statistical analysis (STATA), and was a major contributor in writing manuscript. YL did most parts of statistical analysis (R software), helped interpretation of data. SJL was the other independent database researcher and did parts of quality assessment of the included studies. JG did parts of quality assessment of the included studies and did some data collecting work. YW helped give final decision of the acquisition studies when there were disagreements, analyzed and interpreted the patient data. YL did parts of data colleting work. YZW designed the work, coordinated work arrangement, revised the manuscript and submitted it. All authors read and approved the final manuscript.

Acknowledgements: Not applicable. 


\section{References:}

[1] L.A. Torre, F. Bray, R.L. Siegel, J. Ferlay, J. Lortet-Tieulent, A. Jemal, Global cancer statistics, 2012, CA Cancer J Clin 65(2) (2015) 87-108.

[2] A. Kinoshita, H. Onoda, N. Fushiya, K. Koike, H. Nishino, H. Tajiri, Staging systems for hepatocellular carcinoma: Current status and future perspectives, World J Hepatol 7(3) (2015) 406-24.

[3] J.P. Wedd, E. Nordstrom, T. Nydam, J. Durham, M. Zimmerman, T. Johnson, W. Thomas Purcell, S.W. Biggins, Hepatocellular carcinoma in patients listed for liver transplantation: Current and future allocation policy and management strategies for the individual patient, Liver Transpl 21(12) (2015) $1543-52$.

[4] L.A. Dawson, S. Hashem, A. Bujold, Stereotactic body radiation therapy for hepatocellular carcinoma, Am Soc Clin Oncol Educ Book (2012) 261-4.

[5] M. Scorsetti, T. Comito, L. Cozzi, E. Clerici, A. Tozzi, C. Franzese, P. Navarria, A. Fogliata, S. Tomatis, G. D'Agostino, C. Iftode, P. Mancosu, R. Ceriani, G. Torzilli, The challenge of inoperable hepatocellular carcinoma (HCC): results of a single-institutional experience on stereotactic body radiation therapy (SBRT), J Cancer Res Clin Oncol 141(7) (2015) 1301-9.

[6] S.M. Yoon, Y.S. Lim, M.J. Park, S.Y. Kim, B. Cho, J.H. Shim, K.M. Kim, H.C. Lee, Y.H. Chung, Y.S. Lee, S.G. Lee, Y.S. Lee, J.H. Park, J.H. Kim, Stereotactic body radiation therapy as an alternative treatment for small hepatocellular carcinoma, PLoS One 8(11) (2013) e79854.

[7] J. Sun, T. Zhang, J. Wang, W. Li, A. Zhang, W. He, D. Zhang, D. Li, J. Ding, X. Duan, Biologically effective dose (BED) of stereotactic body radiation therapy (SBRT) was an important factor of therapeutic efficacy in patients with hepatocellular carcinoma $(</=5 \mathrm{~cm}), \mathrm{BMC}$ Cancer $19(1)(2019) 846$.

[8] N.D. Parikh, V.D. Marshall, M. Green, T.S. Lawrence, N. Razumilava, D. Owen, A.G. Singal, M. Feng, Effectiveness and cost of radiofrequency ablation and stereotactic body radiotherapy for treatment of early-stage hepatocellular carcinoma: An analysis of SEER-medicare, J Med Imaging Radiat Oncol 62(5) (2018) 673-681.

[9] K. Shiozawa, M. Watanabe, T. Ikehara, Y. Matsukiyo, M. Kogame, Y. Kishimoto, Y. Okubo, H. Makino, N. Tsukamoto, Y. Igarashi, Y. Sumino, Comparison of percutaneous radiofrequency ablation and CyberKnife((R)) for initial solitary hepatocellular carcinoma: A pilot study, World J Gastroenterol 21(48) (2015) 13490-9.

[10] Z.C. Zeng, J. Seong, S.M. Yoon, J.C. Cheng, K.O. Lam, A.S. Lee, A. Law, J.Y. Zhang, Y. Hu, Consensus on Stereotactic Body Radiation Therapy for SmallSized Hepatocellular Carcinoma at the 7th Asia-Pacific Primary Liver Cancer Expert Meeting, Liver Cancer 6(4) (2017) 264-274.

[11] D.F. Stroup, J.A. Berlin, S.C. Morton, I. Olkin, G.D. Williamson, D. Rennie, D. Moher, B.J. Becker, T.A. Sipe, S.B. Thacker, Meta-analysis of observational studies in epidemiology: a proposal for reporting. Meta-analysis Of Observational Studies in Epidemiology (MOOSE) group, JAMA $283(15)$ (2000) $2008-12$.

[12] J.F. Tierney, L.A. Stewart, D. Ghersi, S. Burdett, M.R. Sydes, Practical methods for incorporating summary time-to-event data into meta-analysis, Trials 8 (2007) 16.

[13] J.H. Kwon, S.H. Bae, J.Y. Kim, B.O. Choi, H.S. Jang, J.W. Jang, J.Y. Choi, S.K. Yoon, K.W. Chung, Long-term effect of stereotactic body radiation therapy for primary hepatocellular carcinoma ineligible for local ablation therapy or surgical resection. Stereotactic radiotherapy for liver cancer, BMC Cancer 10 (2010) 475 .

[14] B.G. Jun, Y.D. Kim, G.J. Cheon, E.S. Kim, E. Jwa, S.G. Kim, Y.S. Kim, B.S. Kim, S.W. Jeong, J.Y. Jang, S.H. Lee, H.S. Kim, Clinical significance of radiationinduced liver disease after stereotactic body radiation therapy for hepatocellular carcinoma, Korean J Intern Med 33(6) (2018) $1093-1102$.

[15] J. Jung, S.M. Yoon, S.Y. Kim, B. Cho, J.H. Park, S.S. Kim, S.Y. Song, S.W. Lee, S.D. Ahn, E.K. Choi, J.H. Kim, Radiation-induced liver disease after stereotactic body radiotherapy for small hepatocellular carcinoma: clinical and dose-volumetric parameters, Radiat Oncol 8 (2013) 249.

[16] Y. Jeong, J. Jung, B. Cho, J. Kwak, C. Jeong, J.H. Kim, J.H. Park, S.Y. Kim, J.H. Shim, K.M. Kim, Y.S. Lim, H.C. Lee, S.M. Yoon, Stereotactic body radiation therapy using a respiratory-gated volumetric-modulated arc therapy technique for small hepatocellular carcinoma, BMC Cancer 18(1) (2018) 416.

[17] S. Balduzzi, G. Rucker, G. Schwarzer, How to perform a meta-analysis with R: a practical tutorial, Evid Based Ment Health 22(4) (2019) $153-160$.

[18] B.G. Jun, S.G. Kim, Y.D. Kim, G.J. Cheon, K.H. Han, J.J. Yoo, Y.S. Kim, S.W. Jeong, J.Y. Jang, S.H. Lee, S. Park, H.S. Kim, Combined therapy of transarterial chemoembolization and stereotactic body radiation therapy versus transarterial chemoembolization for $</=5 \mathrm{~cm}$ hepatocellular carcinoma: Propensity score matching analysis, PLoS One 13(10) (2018) e0206381.

[19] T.S. Su, P. Liang, H.Z. Lu, J. Liang, Y.C. Gao, Y. Zhou, Y. Huang, M.Y. Tang, J.N. Liang, Stereotactic body radiation therapy for small primary or recurrent hepatocellular carcinoma in 132 Chinese patients, J Surg Oncol 113(2) (2016) 181-7.

[20] T. Kimura, H. Aikata, S. Takahashi, I. Takahashi, I. Nishibuchi, Y. Doi, M. Kenjo, Y. Murakami, Y. Honda, H. Kakizawa, K. Awai, K. Chayama, Y. Nagata, Stereotactic body radiotherapy for patients with small hepatocellular carcinoma ineligible for resection or ablation therapies, Hepatol Res 45(4) (2015) 378-86.

[21] N. Sanuki, A. Takeda, Y. Oku, T. Mizuno, Y. Aoki, T. Eriguchi, S. Iwabuchi, E. Kunieda, Stereotactic body radiotherapy for small hepatocellular carcinoma: a retrospective outcome analysis in 185 patients, Acta Oncol 53(3) (2014) 399-404. 
[22] A. Takeda, N. Sanuki, Y. Tsurugai, S. Iwabuchi, K. Matsunaga, H. Ebinuma, K. Imajo, Y. Aoki, H. Saito, E. Kunieda, Phase 2 study of stereotactic body radiotherapy and optional transarterial chemoembolization for solitary hepatocellular carcinoma not amenable to resection and radiofrequency ablation, Cancer 122(13) (2016) 2041-9.

[23] J.M. Llovet, J. Fuster, J. Bruix, Intention-to-treat analysis of surgical treatment for early hepatocellular carcinoma: resection versus transplantation, Hepatology 30(6) (1999) 1434-40.

[24] T. Shibata, H. Isoda, Y. Hirokawa, S. Arizono, K. Shimada, K. Togashi, Small hepatocellular carcinoma: is radiofrequency ablation combined with transcatheter arterial chemoembolization more effective than radiofrequency ablation alone for treatment?, Radiology 252(3) (2009) 905-13.

[25] K.U. Schallreuter, M.A. Gupta, Allergic contact dermatitis from tetrachlordecaoxide (Oxoferin), Contact Dermatitis 17(4) (1987) 253-4.

[26] S. Jonas, W.O. Bechstein, T. Steinmuller, M. Herrmann, C. Radke, T. Berg, U. Settmacher, P. Neuhaus, Vascular invasion and histopathologic grading determine outcome after liver transplantation for hepatocellular carcinoma in cirrhosis, Hepatology 33(5) (2001) 1080-6.

[27] S.A. Shah, S.P. Cleary, J.C. Tan, A.C. Wei, S. Gallinger, D.R. Grant, P.D. Greig, An analysis of resection vs transplantation for early hepatocellular carcinoma: defining the optimal therapy at a single institution, Ann Surg Oncol 14(9) (2007) 2608-14.

[28] C.H. Cha, L. Ruo, Y. Fong, W.R. Jarnagin, J. Shia, L.H. Blumgart, R.P. DeMatteo, Resection of hepatocellular carcinoma in patients otherwise eligible for transplantation, Ann Surg 238(3) (2003) 315-21; discussion 321-3.

[29] R.T. Poon, S.T. Fan, C.M. Lo, C.L. Liu, J. Wong, Long-term survival and pattern of recurrence after resection of small hepatocellular carcinoma in patients with preserved liver function: implications for a strategy of salvage transplantation, Ann Surg 235(3) (2002) 373-82.

[30] M.S. Chen, J.Q. Li, Y. Zheng, R.P. Guo, H.H. Liang, Y.Q. Zhang, X.J. Lin, W.Y. Lau, A prospective randomized trial comparing percutaneous local ablative therapy and partial hepatectomy for small hepatocellular carcinoma, Ann Surg 243(3) (2006) 321-8.

[31] D.H. Lee, J.M. Lee, J.Y. Lee, S.H. Kim, J.H. Yoon, Y.J. Kim, J.K. Han, B.I. Choi, Radiofrequency ablation of hepatocellular carcinoma as first-line treatment: long-term results and prognostic factors in 162 patients with cirrhosis, Radiology 270(3) (2014) 900-9.

[32] M. Dobrzycka, P. Spychalski, O. Rostkowska, M. Wilczynski, P. Kobiela, M. Grat, V. Dell'Acqua, M. Hoyer, B.A. Jereczek-Fossa, Stereotactic body radiation therapy for early-stage hepatocellular carcinoma - a systematic review on outcome, Acta Oncol 58(12) (2019) 1706-1713.

[33] C.H. Rim, H.J. Kim, J. Seong, Clinical feasibility and efficacy of stereotactic body radiotherapy for hepatocellular carcinoma: A systematic review and meta-analysis of observational studies, Radiother Oncol 131 (2019) 135-144.

[34] M. Blettner, W. Sauerbrei, B. Schlehofer, T. Scheuchenpflug, C. Friedenreich, Traditional reviews, meta-analyses and pooled analyses in epidemiology, Int J Epidemiol 28(1) (1999) 1-9.

[35] Wells G, Shea B, O'connell D, Peterson J, Welch V, Losos M, et al. The Newcastle-Ottawa Scale (NOS) for assessing the quality of nonrandomised studies in meta-analyses. oxford: Ottawa Hospital Research Institute; 2014. ASp; 2015.

[36] Cochran WG. The combination of estimates from different experiments. Biometrics 10 (1954) 101-29.

[37] Egger M, Smith GD, Minder C. Bias in meta-analysis detected by a simple, graphical test-Reply. Brit Med J 316 (1998) $470-1$.

[38] Nitin Ohri, Wolfgang A Tomé, Alejandra Méndez Romero, et al. Int J Radiat Oncol Biol Phys S0360-3016(17)(2018) 34525-X.

\section{Figures}




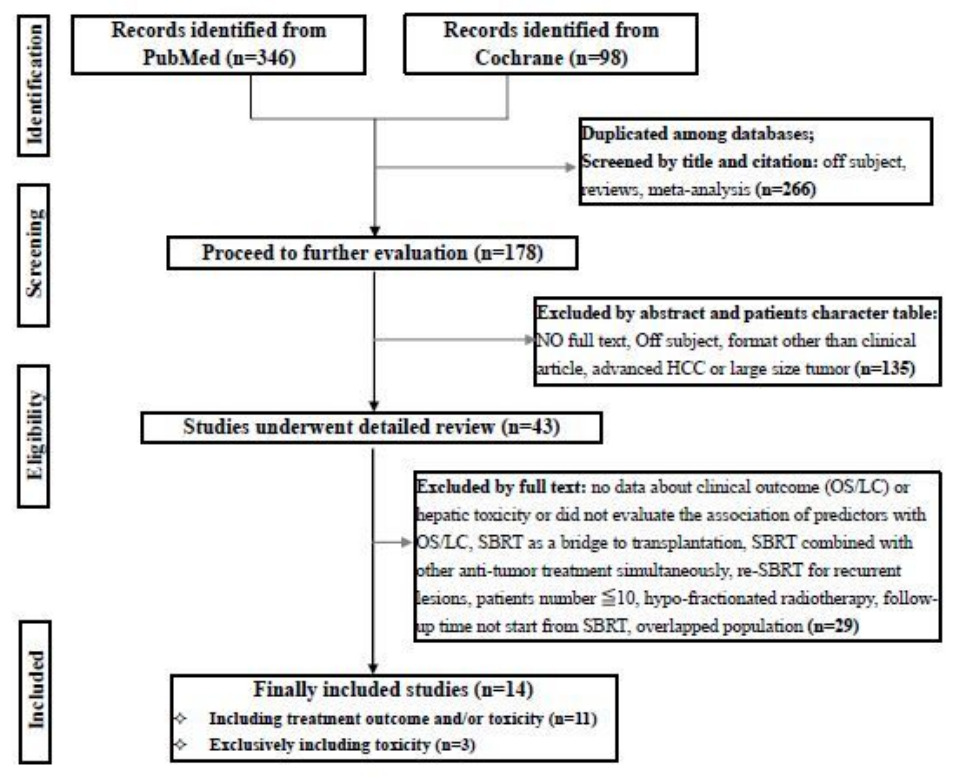

Figure 1. Flowchart of study inclusion

Figure 1

Flowchart of study inclusion

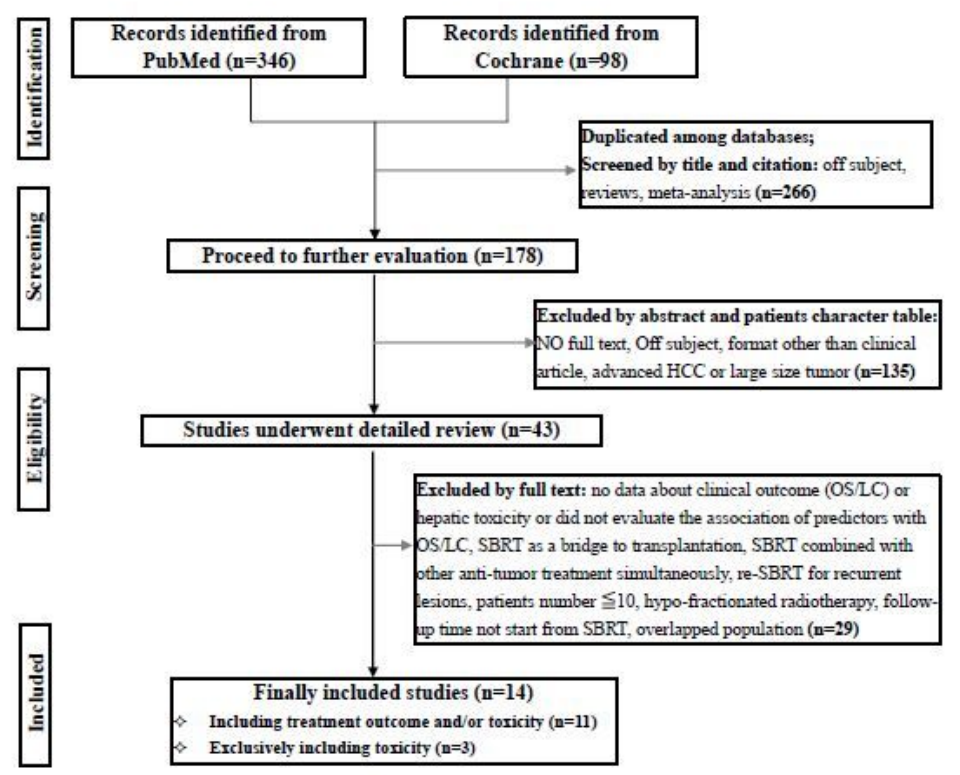

Figure 1. Flowchart of study inclusion

\section{Figure 1}

Flowchart of study inclusion 
a

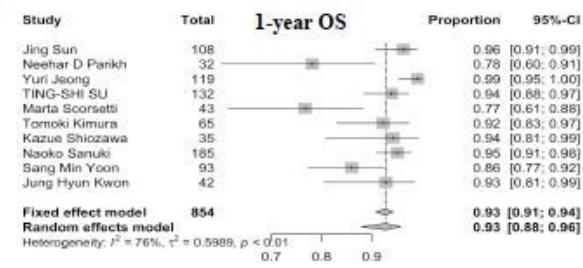

c

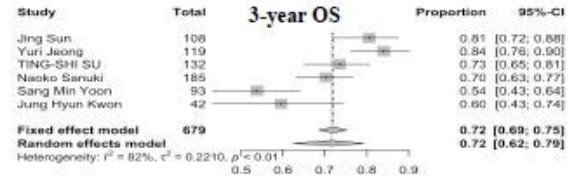

e
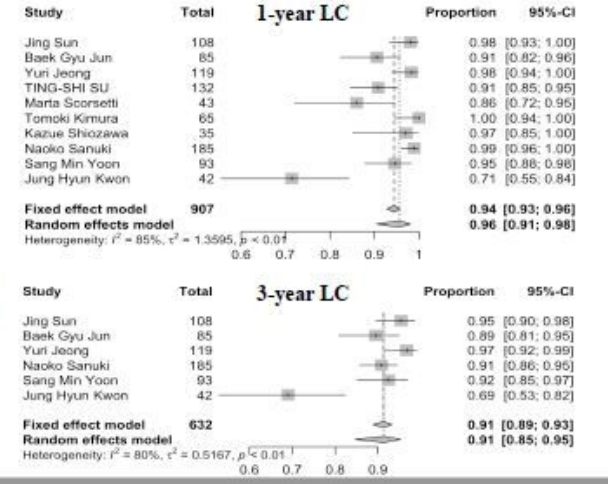

b

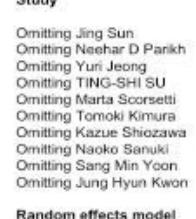

d

Omittin

Omitting Jing Sur Omitiing Yuri SeOng
Omiting TING-SHI SU
Omitting Nacko Sanuk Omitting Nacko Sanuki
Omitting Sang Man Yoon
Omitling Jung Hrun Kwon Random effects model

f study Omitting Jing Sun
Omitting Baek Gyu Ju Omitting Yun Jeong Onitting Marta Scorse Omitting Tomoki Kimura Omitting Kazue Shiozam Omitting Sang Min Yoon
Omitting Jung Hyun Kwo

Random effects model

h

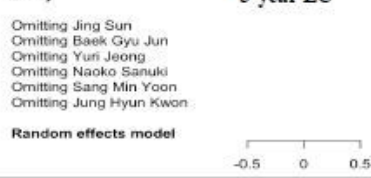

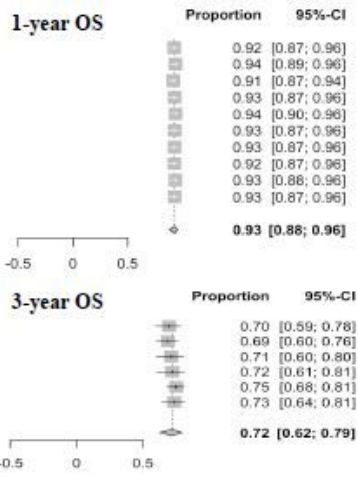

1-year LC

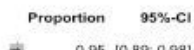

0.95
0.96
$0.89 ; 0.989]$ 0.96 [0.91:0.98]
$0.95[0.89,0.99]$ 0.96 [0.91:098] $0.96[0.91 ; 0.99]$
$0.95[0.89 ; 0.98]$ 0.95
$0.95[0.99 ; 0.989$
0.95 0.95 10.89, 0.981 0.96 [0.93: 0.98 ]

0.96 [0.91; 0.98]
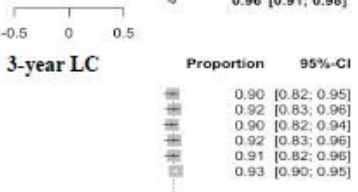

- $\quad 0.91$ [0.85; 0.95]

Figure 2

Forest plots of pooled rates of treatment outcome and sensitive analyses. a-b, pooled 1-year OS rate and related sensitive analyses; c-d, pooled 3-year OS rate and related sensitive analyses; e-f, pooled 1-year LC rate and related sensitive analyses; g-h, pooled 3-year LC rate and related sensitive analyses.

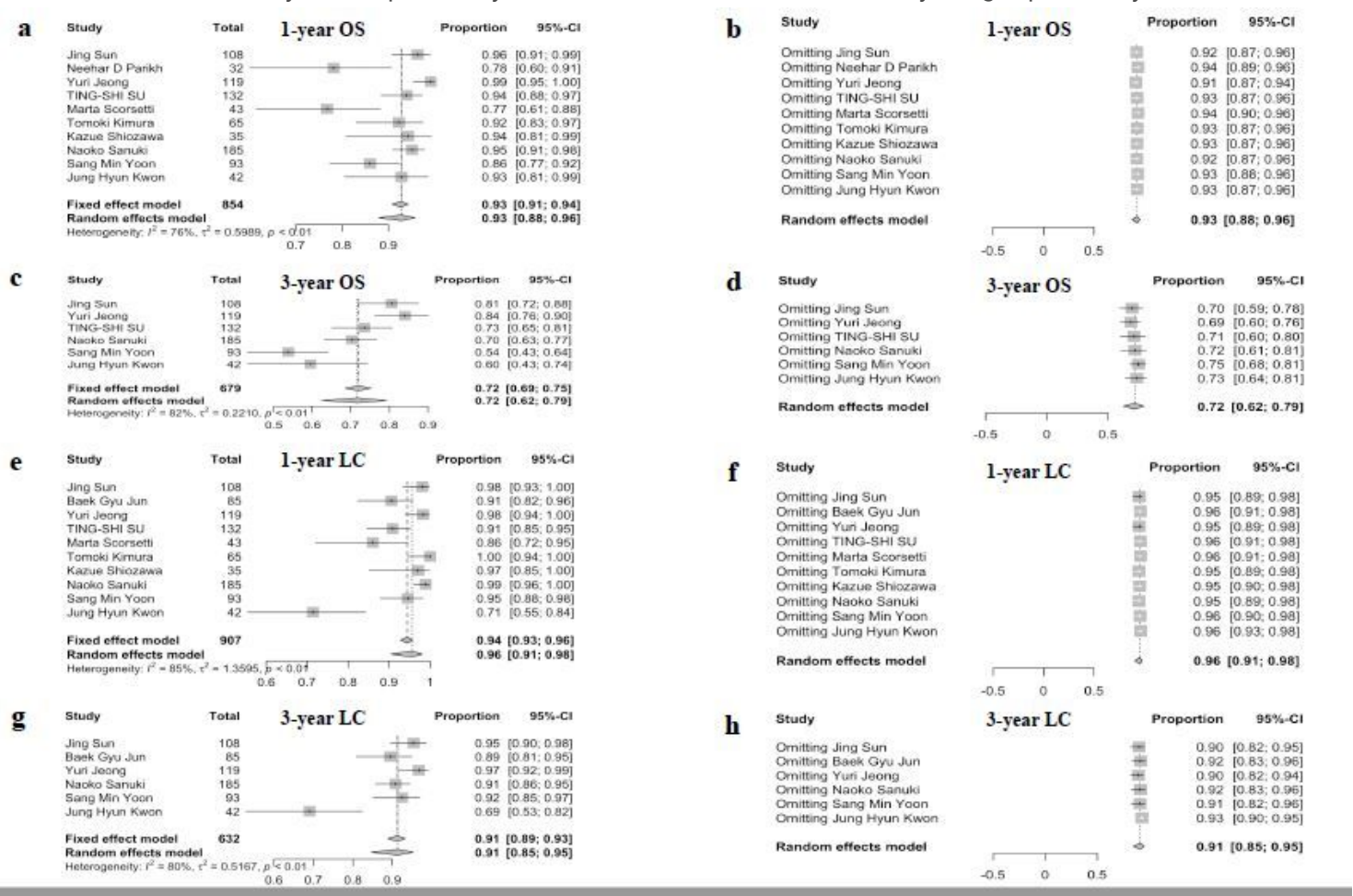

\section{Figure 2}

Forest plots of pooled rates of treatment outcome and sensitive analyses. a-b, pooled 1-year OS rate and related sensitive analyses; c-d, pooled 3-year OS rate and related sensitive analyses; e-f, pooled 1-year LC rate and related sensitive analyses; g-h, pooled 3-year LC rate and related sensitive analyses. 


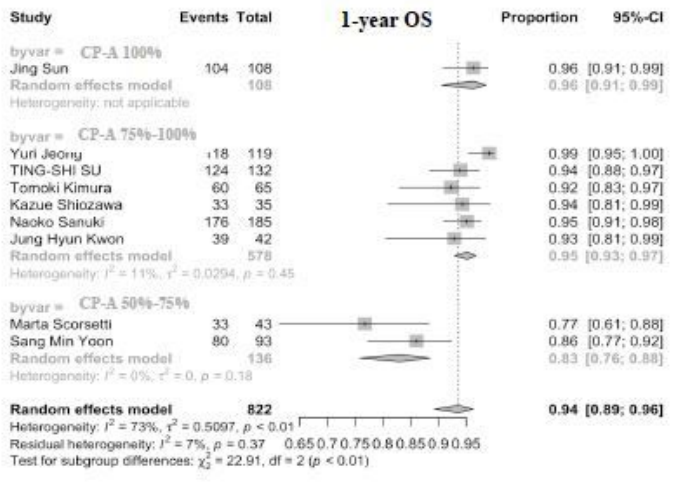

c

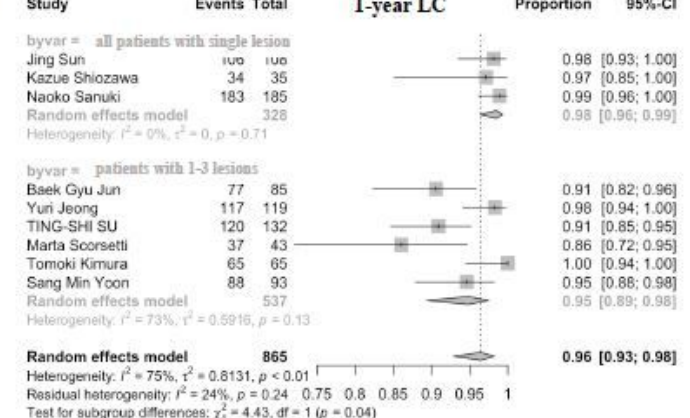

b

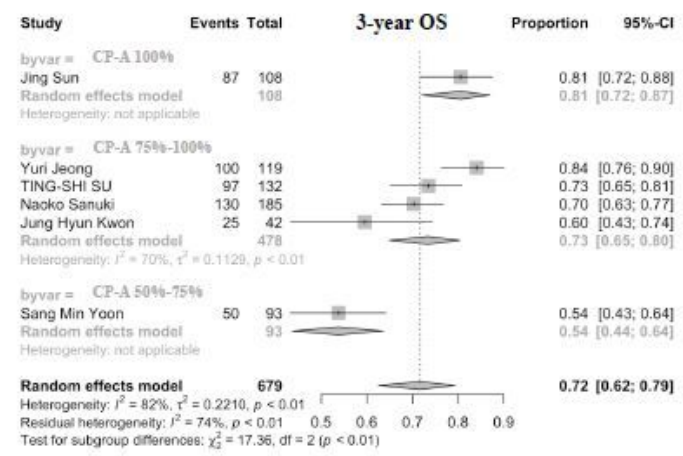

d

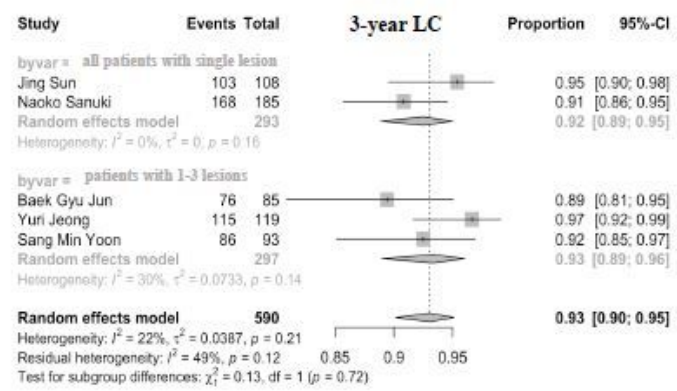

Figure 3

Forest plots of subgroup comparisons for treatment outcome. a, the impact of CP-class on 1-year OS rate; b, the impact of CP-class on 3-year OS; c, the impact of number of lesions on 1-year LC; $d$, the impact of number of lesions on 3-year LC.
a

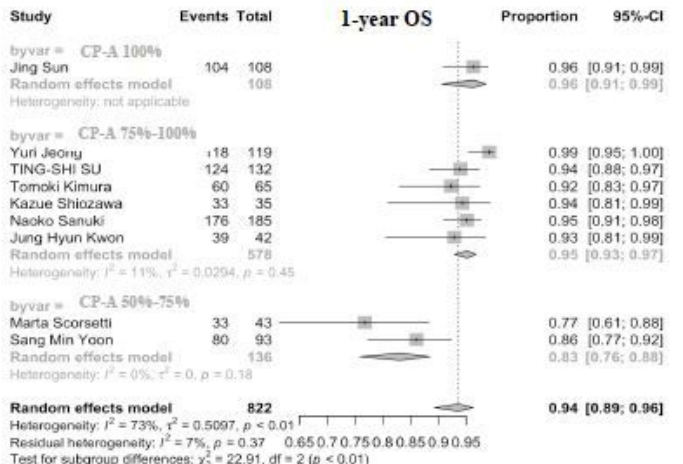
b

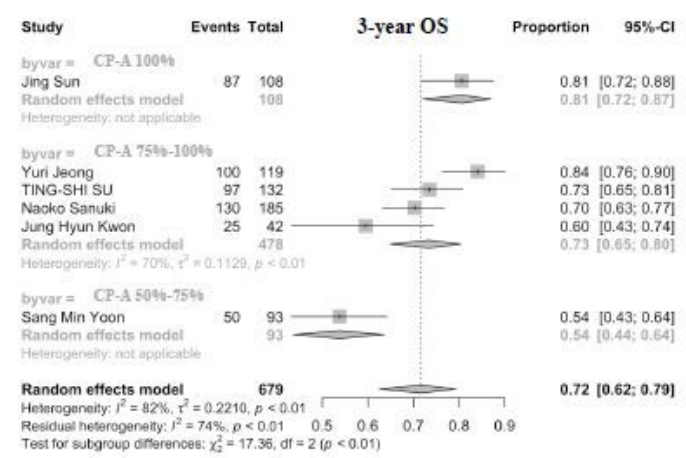
c

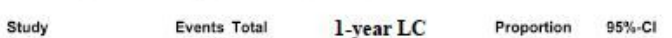
d

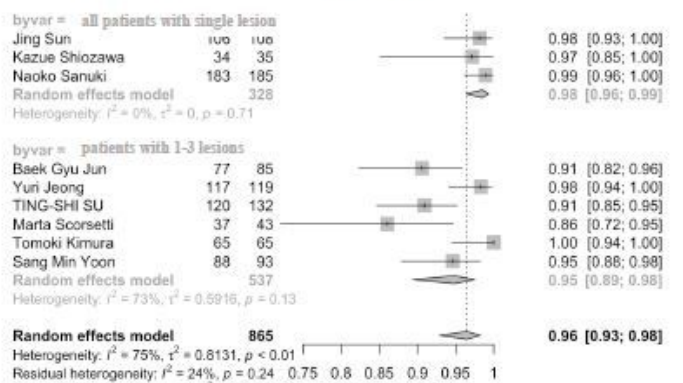

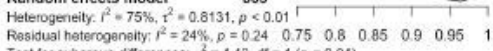
$0.96[0.93 ; 0.98]$ Test lor subgroup dilterences: $x_{1}^{2}=4.43$, df $=1(p=0.04)$

\section{Figure 3}

Forest plots of subgroup comparisons for treatment outcome. a, the impact of CP-class on 1-year OS rate; b, the impact of CP-class on 3-year OS; c, the impact of number of lesions on 1-year LC; $d$, the impact of number of lesions on 3-year LC. 


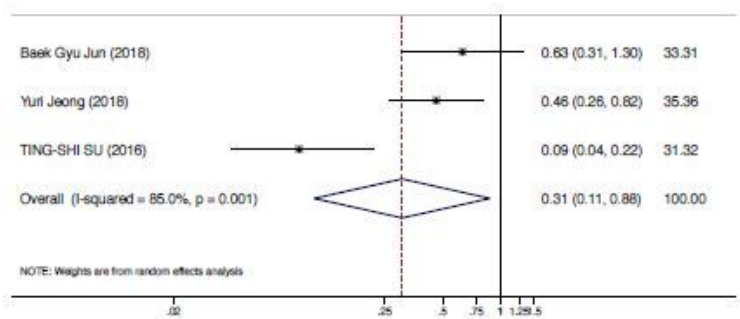

c Age

ID
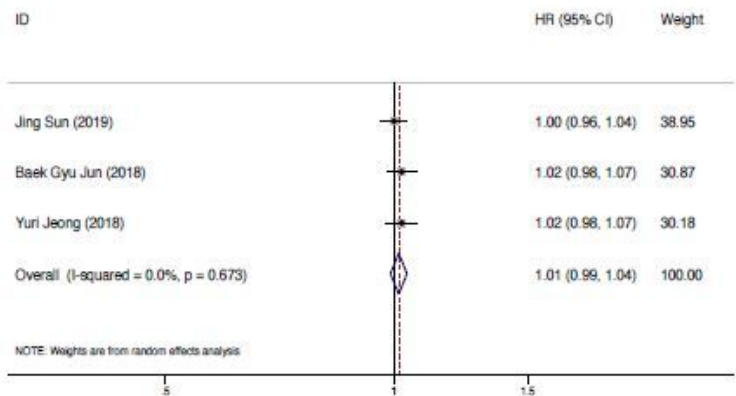

b Tumor size

ID HA $(95 \% \mathrm{Cl})$ Weight

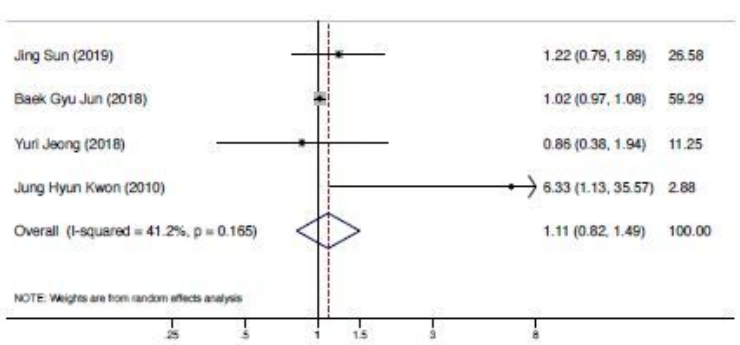

d Sex (Male vs. Female)

HR (95\% Cl) Weight

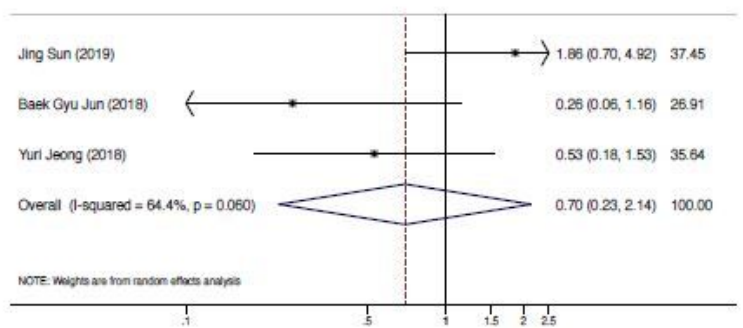

Figure 4

Forest plots of clinical parameters predicting OS in small HCC using SBRT. a, the impact of Child-Pugh class on OS; b, the impact of tumor size (including diameter and volume) on OS; c, the impact of age on OS; $d$, the impact of sex on OS.
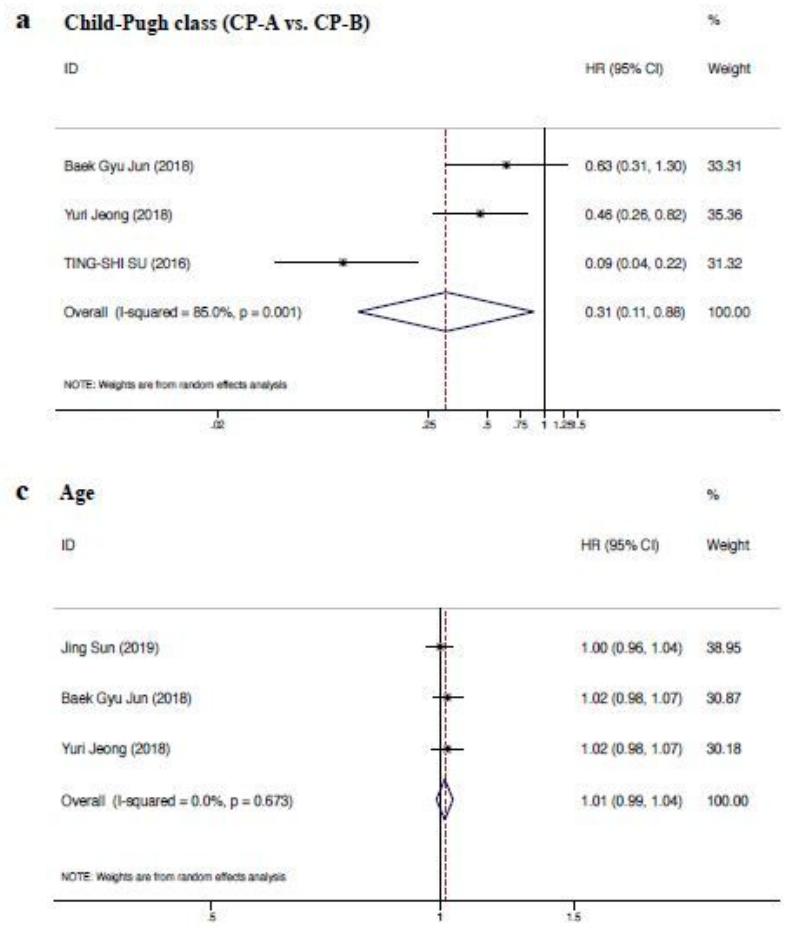

b Tumor size

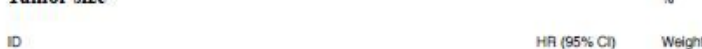

I

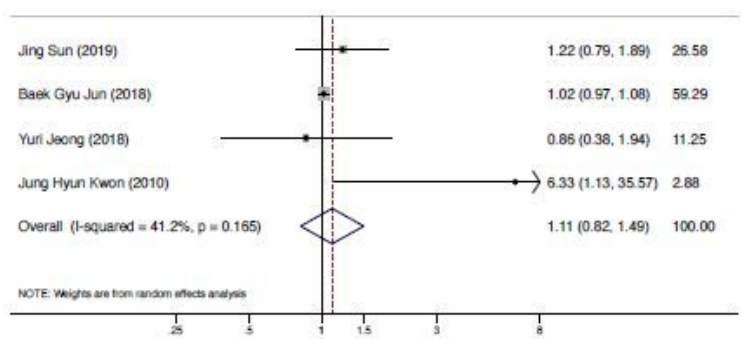

d Sex (Male vs. Female)

10

HP (95\% Cl) Weigh

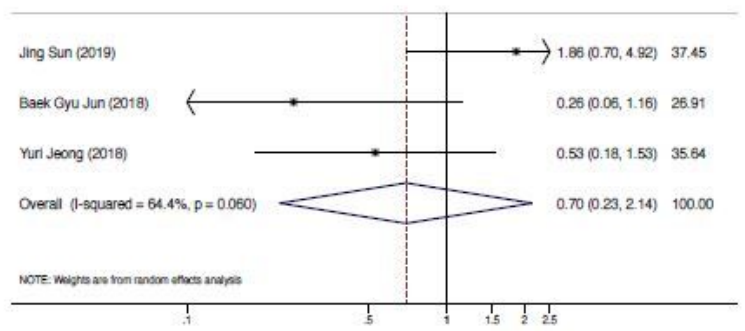

\section{Figure 4}

Forest plots of clinical parameters predicting OS in small HCC using SBRT. a, the impact of Child-Pugh class on OS; b, the impact of tumor size (including diameter and volume) on OS; $c$, the impact of age on OS; $d$, the impact of sex on OS. 
Grade $\geq 3$ hepatic complications

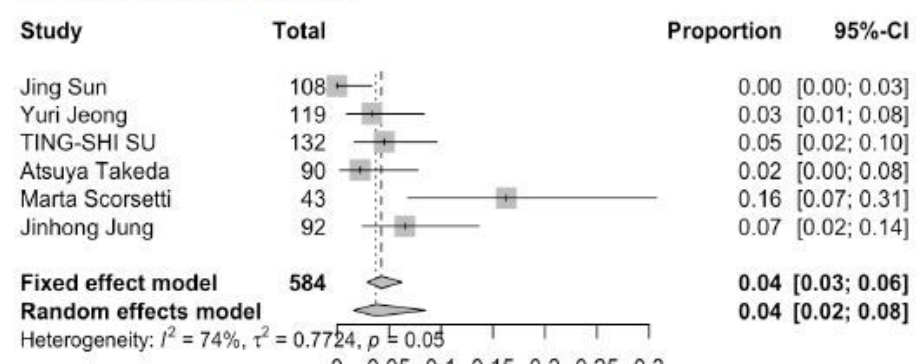

b

RILD

Study

\section{Jing Sun \\ Baek Gyu Jun \\ Yuri Jeong}

Jinhong Jung

Fixed effect model

Random effects model

Heterogeneity: $I^{2}=78 \%, \tau^{2}=0$.

$\begin{array}{lllllll}0 & 0.05 & 0.1 & 0.15 & 0.2 & 0.25 & 0.3\end{array}$

$0.04[0.03 ; 0.06]$
$0.04[0.02 ; 0.08]$

Proportion $\quad 95 \%-\mathrm{Cl}$

$0.07[0.03 ; 0.14]$

$0.25[0.17 ; 0.34]$

$0.08[0.04 ; 0.15]$

$0.18[0.11 ; 0.28]$

$0.15[0.12 ; 0.18]$

$0.14[0.08 ; 0.22]$

Figure 5

Forest plots of pooled rates of hepatic complications in small HCC using SBRT. a, pooled rate of grade $\geq 3$ hepatic complications; b, pooled rate of RILD.

a

Grade $\geq 3$ hepatic complications

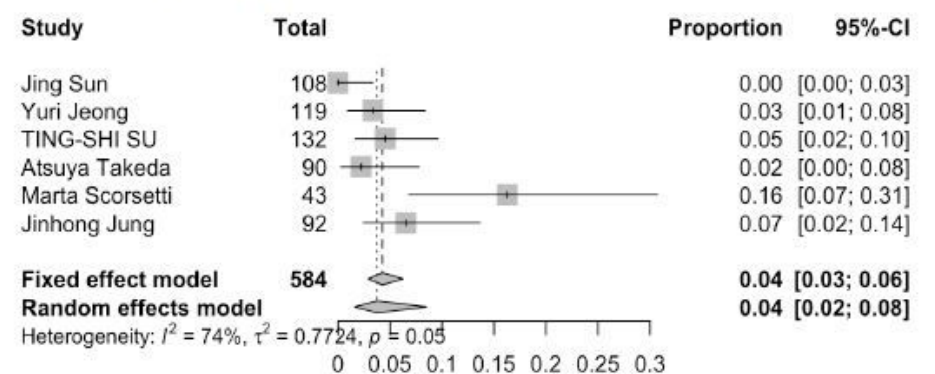

b

RILD

Study

Total

Proportion $\quad 95 \%-\mathrm{Cl}$

Jing Sun

Baek Gyu Jun

Yuri Jeong

Jinhong Jung

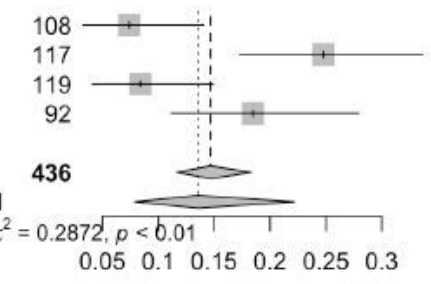

$0.07[0.03 ; 0.14]$

$0.25[0.17 ; 0.34]$

$0.08[0.04 ; 0.15]$

$0.18[0.11 ; 0.28]$

Fixed effect model

Random effects model

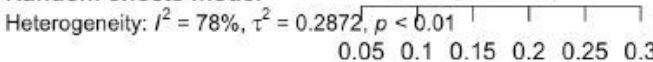

$0.15[0.12 ; 0.18]$

$0.14[0.08 ; 0.22]$

Figure 5

Forest plots of pooled rates of hepatic complications in small HCC using SBRT. a, pooled rate of grade $\geq 3$ hepatic complications; b, pooled rate of RILD. 


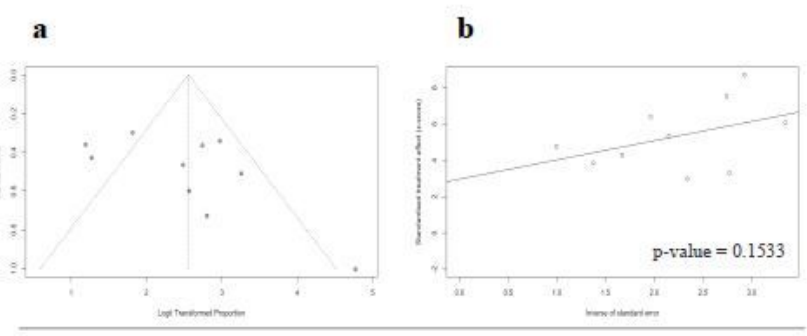

1-year OS
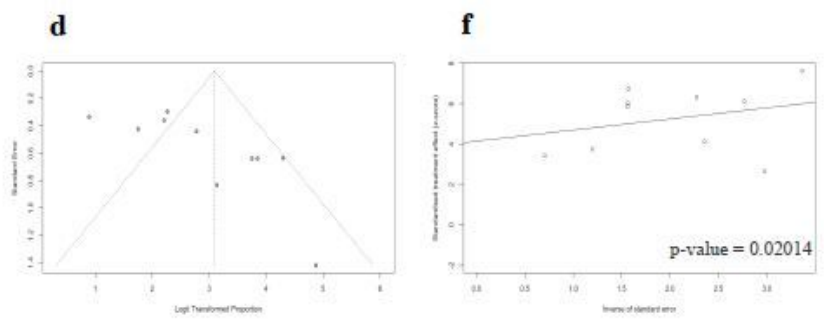

1-year LC
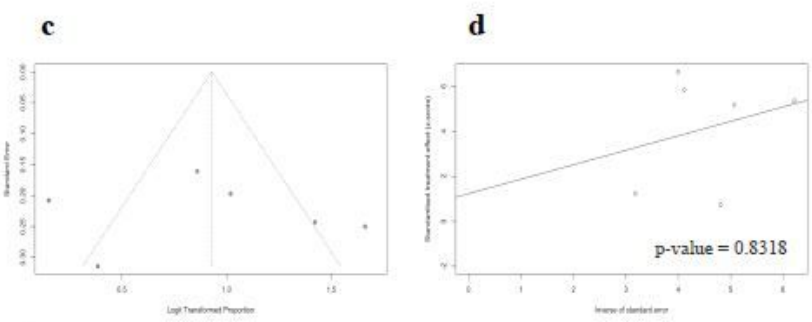

3-year OS
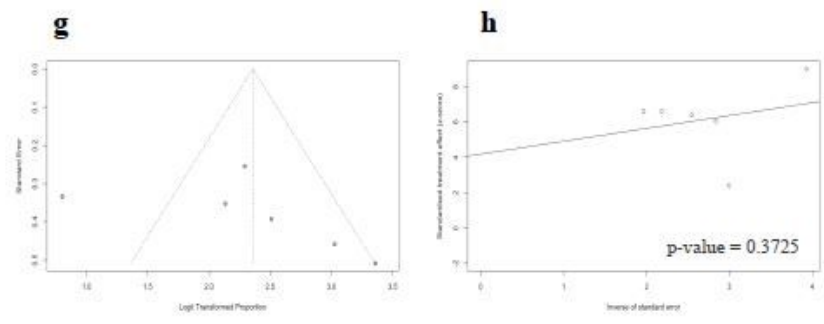

3-year LC

Figure 6

Publication biases of included studies showing in funnel plots and Egger's test.

a

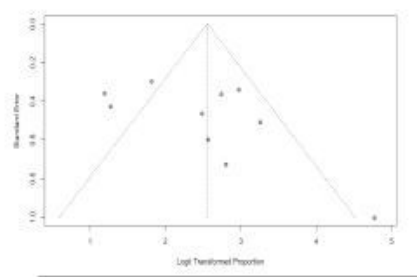

1-year OS

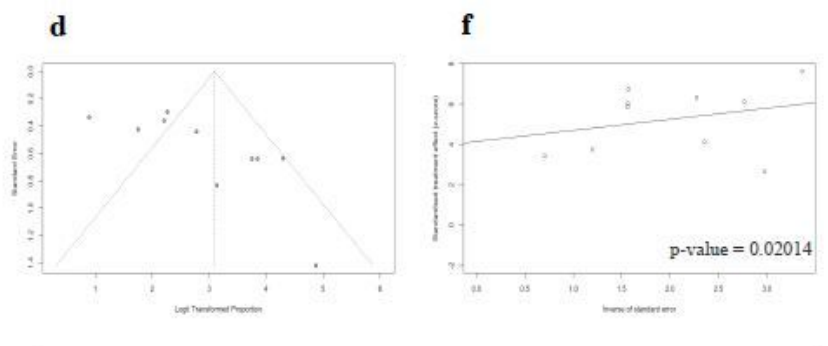

1-year LC b
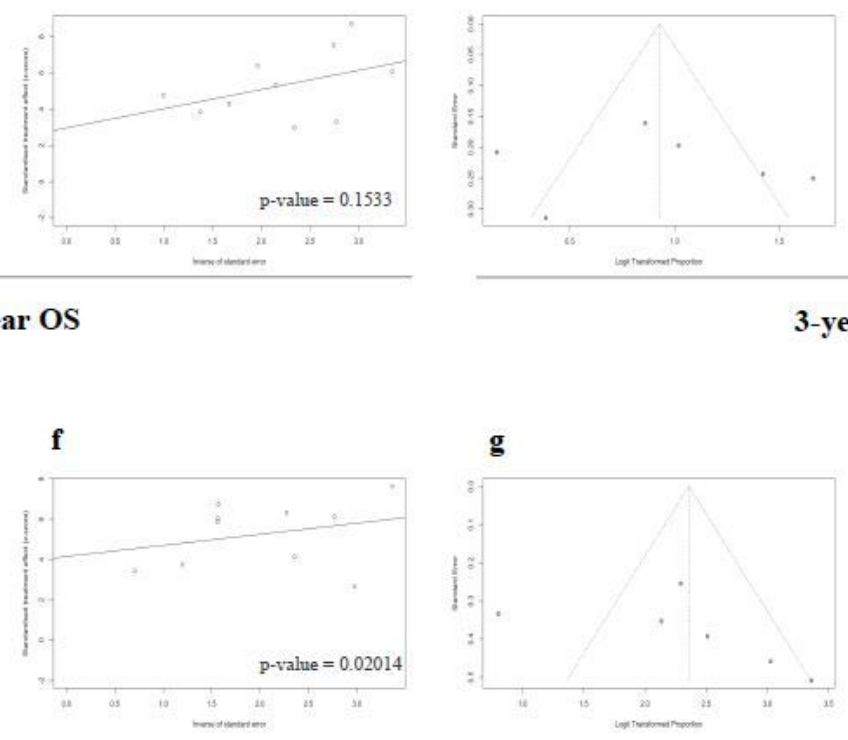

g

d
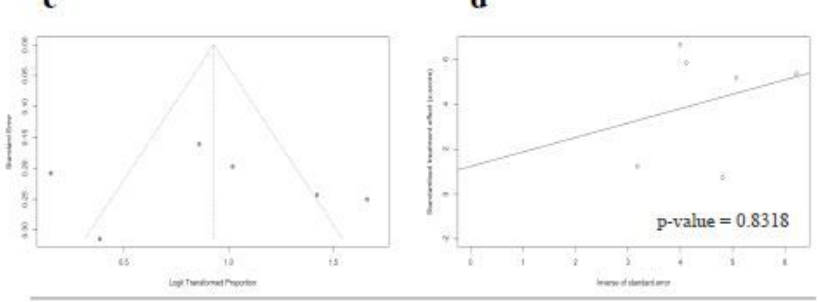

3-year OS

h

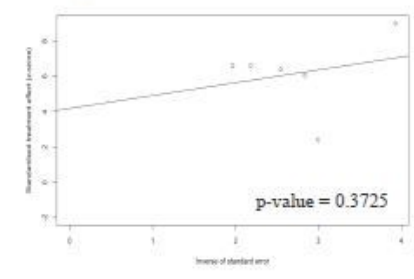

3-year LC

Figure 6

Publication biases of included studies showing in funnel plots and Egger's test.

\section{Supplementary Files}

This is a list of supplementary files associated with this preprint. Click to download.

- datasheet.xlsx

- datasheet.xlsx

- PRISMA2009ChecklistMSWord.doc

- PRISMA2009ChecklistMSWord.doc 
- supplementaryFig.14.pptx

- supplementaryFig.14.pptx

- Supplementarydata1.docx

- Supplementarydata1.docx 